\title{
Interneuron NMDA Receptor Ablation Induces Hippocampus- Prefrontal Cortex Functional Hypoconnectivity after Adolescence in a Mouse Model of Schizophrenia
}

\author{
Rodrigo J. Alvarez, Diego E. Pafundo, Camila L. Zold, and Juan E. Belforte \\ Instituto de Fisiología y Biofísica Bernardo Houssay (IFIBIO-Houssay), Grupo de Neurociencia de Sistemas, Universidad de Buenos Aires y Consejo \\ Nacional de Investigaciones Científicas y Técnicas, Buenos Aires, 1121, Argentina
}

\begin{abstract}
Although the etiology of schizophrenia is still unknown, it is accepted to be a neurodevelopmental disorder that results from the interaction of genetic vulnerabilities and environmental insults. Although schizophrenia's pathophysiology is still unclear, postmortem studies point toward a dysfunction of cortical interneurons as a central element. It has been suggested that alterations in parvalbumin-positive interneurons in schizophrenia are the consequence of a deficient signaling through NMDARs. Animal studies demonstrated that early postnatal ablation of the NMDAR in corticolimbic interneurons induces neurobiochemical, physiological, behavioral, and epidemiological phenotypes related to schizophrenia. Notably, the behavioral abnormalities emerge only after animals complete their maturation during adolescence and are absent if the NMDAR is deleted during adulthood. This suggests that interneuron dysfunction must interact with development to impact on behavior. Here, we assess in vivo how an early NMDAR ablation in corticolimbic interneurons impacts on $\mathrm{mPFC}$ and ventral hippocampus functional connectivity before and after adolescence. In juvenile male mice, NMDAR ablation results in several pathophysiological traits, including increased cortical activity and decreased entrainment to local gamma and distal hippocampal theta rhythms. In addition, adult male KO mice showed reduced ventral hippocampus-mPFC-evoked potentials and an augmented low-frequency stimulation LTD of the pathway, suggesting that there is a functional disconnection between both structures in adult KO mice. Our results demonstrate that early genetic abnormalities in interneurons can interact with postnatal development during adolescence, triggering pathophysiological mechanisms related to schizophrenia that exceed those caused by NMDAR interneuron hypofunction alone.
\end{abstract}

Key words: in vivo electrophysiology; medial PFC; mice; NMDA receptor; parvalbumin interneuron; schizophrenia

Significance Statement:

NMDAR hypofunction in cortical interneurons has been linked to schizophrenia pathophysiology. How a dysfunction of GABAergic cortical interneurons interacts with maturation during adolescence has not been clarified yet. Here, we demonstrate in vivo that early postnatal ablation of the NMDAR in corticolimbic interneurons results in an overactive but desynchronized PFC before adolescence. Final postnatal maturation during this stage outspreads the impact of the genetic manipulation toward a functional disconnection of the ventral hippocampal-prefrontal pathway, probably as a consequence of an exacerbated propensity toward hippocampal-evoked depotentiation plasticity. Our results demonstrate a complex interaction between genetic and developmental factors affecting cortical interneurons and PFC function.

Received Aug. 4, 2019; revised Jan. 7, 2020; accepted Jan. 18, 2020.

Author contributions: R.J.A., D.E.P., C.L.Z., and J.E.B. designed research; R.J.A., D.E.P., and J.E.B. performed research; R.J.A. contributed unpublished reagents/analytic tools; R.J.A. and J.E.B. wrote the first draft of the paper; D.E.P., C.L.Z., and J.E.B. analyzed data; D.E.P., C.L.Z., and J.E.B. edited the paper; C.L.Z. and J.E.B. wrote the paper.

The authors declare no competing financial interests.

This work was supported by National Alliance for Research on Schizophrenia and Depression; Fondo para la Investigación Científica y Tecnológica Grants PICT 2014-0459, PICT 2014-2828, PICT 2016-0724, and PICT 2016-1807; University of Buenos Aires; and Consejo Nacional de Investigaciones Científicas y Técnicas. We thank Jésica Unger, Verónica Risso, and Graciela Ortega for technical assistance; and Kazu Nakazawa for critical reading of the manuscript.

Correspondence should be addressed to Juan E. Belforte at jbelforte@fmed.uba.ar.

https://doi.org/10.1523/JNEUROSCI.1897-19.2020

Copyright $\odot 2020$ the authors

\section{Introduction}

Different perspectives stress the importance of genetic, developmental, and environmental factors in the etiology and pathophysiology of schizophrenia (SZ) (Weinberger, 1987; Tsuang et al., 2001; Lewis and Levitt, 2002; van Os et al., 2010; Brown, 2011; Rapoport et al., 2012). Although a consensus regarding the relative importance of each factor has not yet been reached, it is widely accepted that complex interactions between them are central for the development of the disorder (van Os and Kapur, 2009). For instance, gene-environment interactions have been extensively explored in patients (Tsuang et al., 2001) as well as in SZ animal models (Kannan et al., 2013). Environmental factors 
that alter neurodevelopmental trajectory could be present at any time; however, for SZ, the consensus tends to aggregate them around the perinatal period and during adolescence. Adolescence is a stressful period of life in humans and animals, where significant anatomic, physiological, and behavioral changes take place (Spear, 2000; Casey et al., 2008). Thus, adolescence has even been proposed as a critical period for vulnerability to SZ and other psychiatric disorders (Paus et al., 2008; Hoftman and Lewis, 2011; Do et al., 2015).

Some SZ-related animal models have been extremely useful to unveil pathophysiological changes that emerge during adolescence, but most of them are based on pharmacological (Mouri et al., 2007; Gomes et al., 2016) or lesion-derived manipulations (Tseng et al., 2009) and lack anatomic or genetic specificity. Thus, how normal development during adolescence interacts with genetic susceptibilities is still largely unexplored, in part due to the limited availability of animal models originated in a specific genetic manipulation that include a developmental component for the emergence of SZ phenotypes. We have previously demonstrated that genetic ablation of NMDAR-indispensable subunit Type 1 (GRIN1, encoded by Grin1 gene) in cortical, mostly parvalbumin-positive $\left(\mathrm{PV}^{+}\right)$interneurons, during an early postnatal stage, results in neurochemical, physiological, behavioral, and epidemiological phenotypes compatible with SZ only after mutant mice reach adulthood (Belforte et al., 2010; Jiang et al., 2013; Nakao et al., 2019). Remarkably, we and others have shown that ablation of this glutamatergic receptor in $\mathrm{PV}^{+}$ interneurons during adulthood, instead of early postnatal period, does not result in a SZ-compatible phenotype (Fuchs et al., 2007; Meletis et al., 2011; Pozzi et al., 2014). Since early and adult Grin1-ablated KO mice have the same genotype, the remarkable difference in behavioral phenotypes in adulthood must result from the interaction of the genetic manipulation with physiological developmental changes. Also, in our previous study, we showed that the Grin 1 ablation in young KO is completed before adolescence; we therefore speculate that the SZ-related phenotypes that emerge during adulthood are a consequence of an abnormal maturation occurring during adolescence.

To gain insight on the pathophysiological changes induced by early postnatal Grin1 ablation in cortical and hippocampal $\mathrm{PV}^{+}$ interneurons and its interaction with development, we analyzed several physiological aspects of $\mathrm{mPFC}$ neural activity in vivo before and after adolescence in Grin1 $\mathrm{KO}$ and control mice. We focus our study in the $\mathrm{mPFC}$ due to its clear relation with $\mathrm{SZ}$ symptoms and its protracted maturation that spans adolescence both in humans and rodents. Given that $\mathrm{PV}^{+}$interneurons can be involved in both feedforward and feedback inhibition, we explore the status of firing rate, neuronal synchronization to local and distal cortical rhythms, and distal input processing (i.e., ventral hippocampal [vHP] to mPFC functional connectivity).

We expect that our work may contribute to unveil pathophysiological mechanisms involved in SZ, allowing the identification of putative therapeutics targets based on altered physiology, and not only in altered behavior, in concordance with the approach proposed by the Research Domain Criteria framework (Insel et al., 2010).

\section{Materials and Methods}

\section{Ethics}

All experimental procedures were in accordance with institutional (CICUAL, RS783/15 University of Buenos Aires) and government regulations (SENASA, RS617/2002). All efforts were made to minimize the number of animals used and their suffering.

\section{Animals}

We used male Ppp1r2-Cre/homozygously-floxed-Grin1 KO mice (Grin 1 $\mathrm{KO}$, hereafter mutant or KO mice) and Cre-negative control littermates (homozygously-floxed-Grin1: control). The Grin1 KO mice were generated as previously described (Belforte et al., 2010). This animal model has already been extensively characterized regarding the Cre-mediated expression and recombination pattern in different brain regions, including $\mathrm{mPFC}$ and HP (Belforte et al., 2010; Nakao et al., 2019). Ppp1r2-Cre recombination pattern follows endogenous Ppp1r2 expression pattern, with the important exception of nucleus accumbens and pyramidal layer in CA1 probably as a consequence of its transgenic nature. Also, in our previous publication, Ppp1r2-Cre line was tested in a number of behavioral tasks and neurochemical and electrophysiological aspects showing no significant differences with control mice. In this line, genetic deletion of obligatory NMDA Grin1 subunit occurs in $~ 50 \%$ of cortical and hippocampal GABAergic interneurons, including $>75 \%$ of $\mathrm{PV}^{+}$cells, from the second postnatal week (Grin1 KO mice). Stable Cre-mediated recombination was previously observed from postnatal week 4 to week 20. Therefore, preadolescent mice were studied between postnatal days 28 and 31 (hereafter juvenile mice), whereas 12- to 16-week-old mice constitute the adult group. All mice were maintained in C57BL/6NTac background by crossing to C57BL/6NTac (B6) strain seven times. The female Grin1 KO mice were further crossed to homozygous-floxed Grin 1 males to produce next-generation mutants and control homozygous-floxed Grin1 littermate mice. The genotype was identified by PCR using tail DNA obtained after the recording session for each mouse. All animals were maintained under a 12:12 h dark/light cycle with food and water ad libitium. Pups were weaned between 21 and $28 \mathrm{~d}$ of age and group-housed in same-sex sibling pairs until recordings were made. The previous neurobiological, behavioral, and physiological validation of the model has been conducted in male mice; therefore, for comparative purposes, we have focused our analysis in males.

\section{General in vivo electrophysiological procedures}

All recordings were performed under urethane anesthesia $(1.6 \times \mathrm{g} / \mathrm{kg}$ in saline, i.p., Sigma Millipore U2500) following published protocols (de Almeida et al., 2013). Mice were also treated with a local anesthetic in the scalp and pressure points (bupivacaine hydrochlorate solution, 5\% wv/v, Durocaine, AstraZeneca, $0.1-0.3 \mathrm{ml}$ s.c.) and secured to a stereotaxic frame (Stoelting). Temperature was maintained between $36^{\circ} \mathrm{C}$ and $37^{\circ} \mathrm{C}$ with a servo-controlled heating pad. Additional urethane was administered as required to obtain a stable anesthesia level assessed by reflex response to a tail pinch and online visual examination of the frontal cortex electrocorticogram (customarily $0.1-0.2 \times g / \mathrm{kg}$ i.p. every $1-3$ h) (de Almeida et al., 2013; Braz et al., 2015). Small craniotomies were drilled above the target regions according to each experimental condition. Urethane was chosen since it provides a constant level of anesthesia over several hours providing a homogeneous background state, and a stereotyped pattern of cortical activity similar in both experimental ages (de Almeida et al., 2013). Under urethane anesthesia, the EEG alternates between two global brain states: (1) a prevalent slow wave state, with coordinated $\delta$ oscillatory activity across the cortex resembling natural slow wave sleep; and (2) periods of desynchronized state, with enhanced high-frequency components resembling wakefulness EEG (Gervasoni et al., 2004; Clement et al., 2008; Kasanetz et al., 2008) and associated with a strong theta activity in local field potential (LFP) from vHP (Fig. 1C; "desynchronized state"). Custom-made software allowed automatic selection of epochs with low slow wave power $(0.5-2 \mathrm{~Hz})$ in frontal cortex and high theta relative power $(3-6 \mathrm{~Hz})$ in the vHP, similarly as described previously (de Almeida et al., 2013). For this study, we selected urethane as an anesthetic since it has been reported to produce minimal disruption of physiologically relevant measures (Maggi and Meli, 1986). Even more, it has been proposed that urethane promotes a condition of behavioral unconsciousness that closely mimics the full spectrum of natural sleep (Clement et al., 2008). Importantly, cortical physiology under desynchronized state in anesthetized animals is similar to wakefulness (Timofeev et al., 2000; Steriade et al., 2001); therefore, all neuronal data presented here correspond exclusively to desynchronized state periods. 

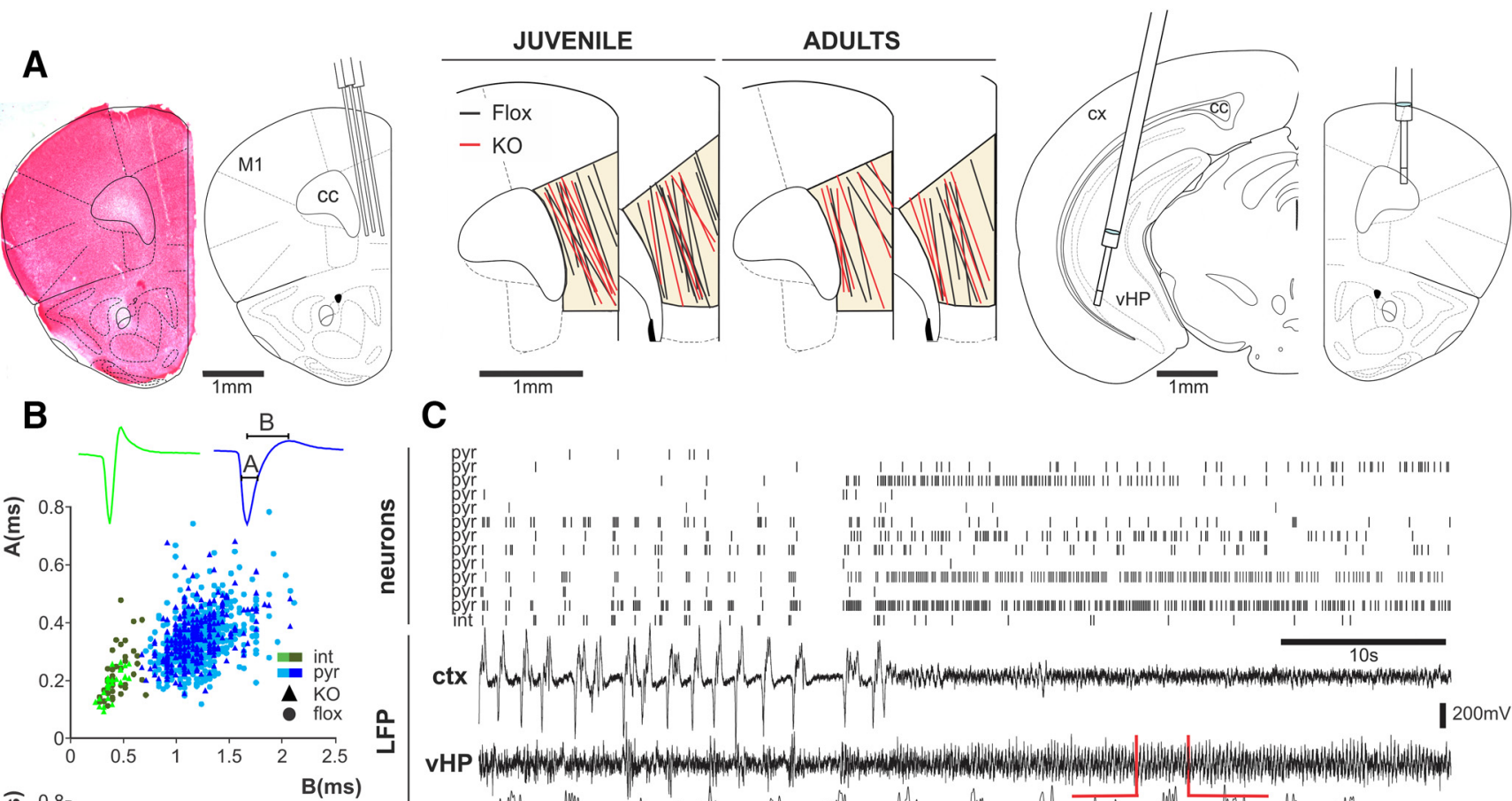

C

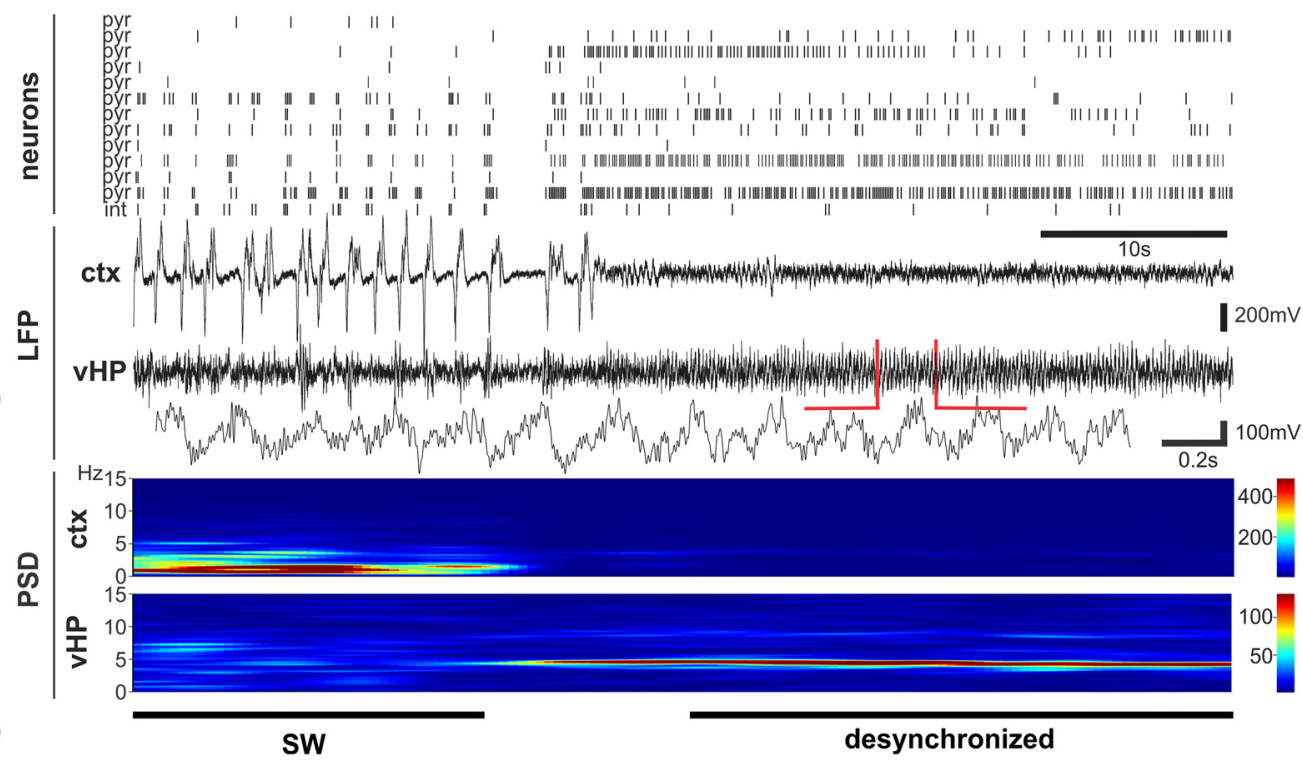

Figure 1. Simultaneously recording of unit activity in $\mathrm{MPFC}$ and LFP in cortex and hippocampus allows separation of putative neocortical interneurons and pyramidal cells during different global brain states. $\boldsymbol{A}$, Left, Safranin 0 staining shows an example of the electrode track left by a tetrode recording in the $\mathrm{mPFC}$, and schematic of a coronal section shows tetrode positioning in the mPFC. Middle, Schematic of coronal sections showing all electrode tracks in $\mathrm{MPFC}$ in juvenile (left) and adult (right) mice. mPFC recordings were performed along mPFC dorsoventral axis. No differences in electrode track positioning were observed across control (Flox, black lines) and KO (red lines) mice. Right, Schematic of coronal sections showing the localization of the concentric bipolar electrodes used to record the VHP LFP (left) and the cortical field potential (right). M1, Motor cortex; $c c$, corpus callosum; $c x$, cortex. B, mPFC pyramidal cell and interneuron classification based on their electrophysiological properties. Average waveforms of isolated units classified as interneuron (green) and pyramidal (blue), including temporal parameters used in the classification process (A, half-amplitude duration; B, trough to peak time). The parameters A and B were plotted for each cell, and two clear clusters were formed and used to sort neurons into pyramidal and interneurons in juvenile (top) and adult mice (bottom). C, Top, Representative raster plot of mPFC neurons (pyramidal and interneurons) recorded simultaneously with the cortical (ctx) and hippocampal (vHP) LFP activity. Bottom, Power density analysis performed on the same signal showing slow wave (SW) activity in the cortical LFP and a spontaneous desynchronization that correlates with the emergence of theta oscillations in the vHP.

\section{Tetrode unitary recordings}

We performed in vivo tetrode recordings from mPFC as previously described (Espíndola et al., 2018) with some modifications. Briefly, an array of three tetrodes (made of twisted $12-\mu \mathrm{m}$ Formvar-insulated Nichrome wire, final impedance adjusted by gold plating to 200-500 $\mathrm{kOhms}$ ) separated $150-250 \mu \mathrm{m}$ in a triangular shape was stereotaxically advanced through the mPFC. The center of the array was inserted with an angle of $10^{\circ}$ in the coronal plane $1.9 \mathrm{~mm}$ anterior to bregma, $0.5 \mathrm{~mm}$ lateral to midline, and was descended $1 \mathrm{~mm}$ from cortical surface by using an hydraulic micromanipulator. To allow tissue stabilization, recordings were made after a resting period of $30 \mathrm{~min}$. Cortical and vHP LFP activities were simultaneously recorded (Fig. $1 A$ ) through two concentric bipolar electrodes (SNE-100, Better Hospital Equipment) positioned at $1.9 \mathrm{~mm}$ anterior to bregma, $1.4 \mathrm{~mm}$ from midline (contralateral to tetrode array) and $1 \mathrm{~mm}$ from cortical surface (frontal cortex) and $3 \mathrm{~mm}$ posterior to bregma, $2.8 \mathrm{~mm}$ from midline (ipsilateral to tetrode array), and $3.7 \mathrm{~mm}$ from skull surface with an angle of $20^{\circ}$ in the coronal plane (vHP). In addition, a ground wire was implanted on the right occipital bone. The microarray and bipolar electrodes were directly connected, via an EIB-27- Micro board (Neuralynx), to a multichannel amplifier chip (RHA2000-EVAL board, Intan Technologies) where signals were filtered (bandwidth tetrodes $0.5-6000 \mathrm{~Hz}$; bipolar electrodes: $0.5-300 \mathrm{~Hz}$ ), digitized, and acquired at a sampling rate of $25 \mathrm{kHz}$ per channel. Spikes were detected, after high-pass filtering of the signal (median filter, half window length: $2.4 \mathrm{~ms}$ ), by thresholding (40 $\mu \mathrm{V}$ ) using NDManager (part of the published free-software package also including Klusters and NeuroScope) (Hazan et al., 2006). Subsequently, spikes were assigned to individual units ("neurons") by semiautomatic spike sorting based on spike's relative amplitudes across four recording wires of a tetrode and PCA (Klusters software). Semiautomatic verification of clusters quality was performed using autocorrelograms, average spikes' shape, and correlation matrixes (Klusters) by a trained operator blinded to the genotype of the animals. The stationarity of signals and the stability of unit isolation were visually verified for each recording session. Recording sessions in which cluster stability could not be confirmed were excluded from our dataset. Neurons were only included in our dataset if: (1) the mean probability of their spikes belonging to any other cluster was $<5 \%$ ( $p<0.05$ error matrix value for cluster similarities, Klusters software); or (2) if they show a clear refractory period in the autocorrelogram $(<0.5 \%$ events in the $\pm 3 \mathrm{~ms}$ bins). All isolated units were divided into two subclasses (putative pyramidal cells and 
interneurons) based on waveform and firing characteristics as published previously (Bartho et al., 2004). In general, putative pyramidal cells were defined as cells with relatively broad spike waveforms (and with negative curvilinear shapes in the afterhyperpolarization phase; Fig. $1 B$ ), whereas putative interneurons had relatively narrow waveforms with positive curvilinear shapes (Belforte et al., 2010). Three cells from the control group and one from a $\mathrm{KO}$ mouse were excluded from the analysis based on inconsistences in the classification process; the results are not significantly affected by this exclusion.

For every animal, we systematically mapped 5 or 6 sites along the vertical axis of the mPFC. On each recording site, tissue was allowed to stabilize for $30 \mathrm{~min}$, and 30-40 min of spontaneous activity was recorded every $300-500 \mu \mathrm{m}$ along the mPFC. At least $15 \mathrm{~min}$ of neuronal activity during desynchronized state was analyzed for each animal.

\section{Analysis of tetrode recordings}

To determine the consequences of NMDAR deletion in cortical and hippocampal $\mathrm{PV}^{+}$interneurons, we calculated the mean firing rates of putative pyramidal cells and interneurons during desynchronized states (Neuroexplorer, Nex Technologies). To determine the relationship between the spiking of mPFC neurons and LFP rhythms (local hippocampal theta rhythms and local mPFC $\gamma$ oscillations), we used a phaselocking analysis. LFP signals from mPFC tetrodes and vHP bipolar electrodes were digitally filtered using custom-made MATLAB routines (zero phase-lag Butterworth digital filter) to obtain the following bandpassed waveforms: 4-7 (theta), 30-48 (low $\gamma$ ), and 52-80 Hz (high $\gamma$ ). A Hilbert transform was used to obtain the phase angle at every point of each waveform ("instantaneous phase"). Next, the number of spikes occurring at different phase angles of each waveform was depicted in circular plots (bin size: $10^{\circ}$ ). Phase-locking of spike discharges to a given frequency band was determined by assessing deviation from uniformity in these circular plots with the Rayleigh test. The strength of synchronization was further assessed by comparing the module of the resultant vectors summarizing these circular distributions as described by de Almeida et al. (2013). To guarantee sufficient statistical power, only neurons that fired at least 50 spikes during desynchronized state were evaluated for the phase-locking analysis. Power spectrum analysis of LFPs signals was calculated using Neuroexplorer. Spike-triggered averages (STAs) are computed by averaging LFP segments from time. Any components of the LFP that are not consistently phase-locked to the spikes are averaged out and are not visible in the STA. As the STA is calculated by summing all LFP segments and then dividing by the number of spikes, the STA is normalized for spike number.

\section{Functional connectivity and plasticity}

In order to evaluate the functional connectivity of the vHP-mPFC pathway, field potential activity was recorded from $\mathrm{mPFC}$ using a monopolar electrode (inner contact of a concentric bipolar electrode SNE-100, Better Hospital Equipment) referenced to a screw in the contralateral occipital bone. Signal was amplified (differential amplifier Akonic Lab1, Akonic), filtered $1 \mathrm{~Hz}$ to $3 \mathrm{kHz}$, and digitalized ( $20 \mathrm{kHz}$, Digidata 1200 , Molecular Devices). The electrode was positioned in the mPFC with an angle of $10^{\circ}$ in the coronal plane ( $1.9 \mathrm{~mm}$ anterior to bregma, $0.5 \mathrm{~mm}$ lateral to midline, $3.3 \mathrm{~mm}$ ventral to the cranium surface); vHP and frontal cortex bipolar electrodes were inserted as described above in tetrode experiments. Single-constant current pulses to the vHP (CA1/subicular region) were delivered by an isolation unit and governed by computercontrolled pulse generator (Master 8 Stimulator, AMPI). Compatible with a monosynaptic response, vHP stimulation evoked short latency negative/positive field potentials in the mPFC (13-16 ms to negative peak) (Thierry et al., 2000). The position of recording and stimulating electrodes was adjusted to maximize the amplitude of the evoked response. Next, constant current pulses of increasing amplitude (50$800 \mu \mathrm{A}, 100 \mu$ s duration, 10 repetitions of each) were delivered every 20 $\mathrm{s}$ to the vHP. At this intensity range, the field potential is most likely to reflect summated excitatory postsynaptic potentials (Laroche et al., 1990). Evoked responses in mPFC were offline filtered $(5 \mathrm{~Hz}$ to $600 \mathrm{~Hz}$, eight-pole Bessel) and peak-to-peak amplitude of individual responses measured using Clampfit 10 Data Analysis Module (Axon). For each animal, stimulus-response curves were constructed by averaging data obtained for each stimulation intensity. After obtaining stimulusresponse curves, two independent subsets of mice were subjected to the following: (1) a low-frequency stimulation protocol to induce LTD (LTD-LFS) in the vHP-mPFC pathway (modified from Caballero et al., 2014a,b); and (2) a high-frequency stimulation protocol to induce LTP (HFS-LTP), similarly to Gurden et al. (2000). All experiments were performed after $30 \mathrm{~min}$ of tissue recovery time. In LTD experiments, baseline responses were recorded during $30 \mathrm{~min}$ by delivering constant current tests stimuli $(100 \mu \mathrm{s})$ every $20 \mathrm{~s}$. Stimulus intensity was set to evoke $70 \%-80 \%$ of the maximal stimulus-response curve observed response. LTD-LFS was induced by 1500 stimulus trains (5 pulses of 100 $\mu \mathrm{s}$ at $250 \mathrm{~Hz}$ at test stimulation intensity) at $1 \mathrm{~Hz}$. After LFS, single-pulse, test stimuli were delivered every $20 \mathrm{~s}$ for another $30-60 \mathrm{~min}$. LTP protocol followed the same schedule, but two series of 10 HFS trains (50 pulses of $100 \mu$ s at $250 \mathrm{~Hz}$ ) at $0.1 \mathrm{~Hz}$ at $10 \mathrm{~min}$ intervals, using the current intensity that generated the minimal response, were applied after baseline measurement. LTD-LFS and HFS-LTP protocols were repeated four consecutive times to assess LTD and LTP saturation. Individual values obtained from test stimuli were normalized against mean baseline and averaged every $6 \mathrm{~min}$.

\section{Slice physiology}

Experiments were conducted in brain slices prepared from the mPFC of 20- to 24-week-old mice. These mice were injected with adeno-associated virus (AAV) particles 13-14 weeks before experiments. An AAV vector tagged with enhanced yellow fluorescent protein (eYFP) and expressing channel rhodopsin (ChR2) under the CaMKII promoter (AAV-CaMKIIa-hChR2(H134R)-EYFP, Therapy Center Vector Core, University of North Carolina) was injected into either the left or right vHP under deep surgical anesthesia (1\% isoflurane) as described previously (Keifman et al., 2019). Briefly, animals were mounted on a stereotaxic frame (Kopf Instruments), and two $0.5 \mu \mathrm{l}$ injections of AAV $\left(2.46 \times 10^{13}\right.$ viral particles per $\left.\mathrm{ml}\right)$ at $0.1 \mu \mathrm{l} / \mathrm{min}$ were performed at the following coordinates: $3.3 \mathrm{~mm}$ posterior to bregma, 3.3 and $3.2 \mathrm{~mm}$ lateral from midline, and 3.2 and $2.7 \mathrm{~mm}$ below cortex surface. Thirteen to 14 weeks after AAV injection, mice were deeply anaesthetized with isoflurane and decapitated. Brains were quickly removed and immersed in ice-cold slicing solution containing $210 \mathrm{~mm}$ sucrose, $10 \mathrm{~mm} \mathrm{NaCl}, 1.9$ mм KCl, $1.2 \mathrm{~mm} \mathrm{Na} \mathrm{HPO}_{4}, 33 \mathrm{~mm} \mathrm{NaHCO}$, $6 \mathrm{~mm} \mathrm{MgCl}, 1 \mathrm{~mm} \mathrm{CaCl}$, and $10 \mathrm{~mm}$ glucose, $\mathrm{pH}$ 7.3-7.4 when bubbled with $95 \% \mathrm{O}_{2}$ and $5 \% \mathrm{CO}_{2}$; $300 \mu \mathrm{m}$ coronal slices of the $\mathrm{mPFC}$ and the vHP were sectioned using a vibrating microtome (Pelco 1000, Ted Pella). Slices containing the vHP were fixed in PFA (4\% in PBS) and mounted for EYFP fluorescence images, and slices containing the $\mathrm{mPFC}$ were immediately placed in an incubation chamber filled with ACSF maintained at $36^{\circ} \mathrm{C}$ and containing $125 \mathrm{~mm} \mathrm{NaCl}, 2.5 \mathrm{~mm} \mathrm{KCl}, 1.25 \mathrm{~mm} \mathrm{Na} \mathrm{HPO}_{4}, 10 \mathrm{~mm}$ glucose, $25 \mathrm{~mm}$ $\mathrm{NaHCO}_{3}, 0.4 \mathrm{~mm}$ ascorbate, $1 \mathrm{~mm} \mathrm{MgCl}_{2}$, and $2 \mathrm{~mm} \mathrm{CaCl}_{2}, \mathrm{pH}$ 7.3-7.4 when gassed with $95 \% \mathrm{O}_{2}$ and $5 \% \mathrm{CO}_{2}$. After 5 min incubation at $36^{\circ} \mathrm{C}$, brain slices were stabilized at room temperature in the same solution for at least $30 \mathrm{~min}$ before they were transferred to the recording chamber. For recording, slices were transferred to a submersion chamber and superfused at $2 \mathrm{ml} \mathrm{min}^{-1}$ with oxygenated ACSF at $30^{\circ} \mathrm{C}-32^{\circ} \mathrm{C}$. Wholecell recordings were obtained from visually identified pyramidal neurons in layer 3 of the mPFC using a Nikon microscope equipped with IR-DIC optics similarly as described previously (Pafundo et al., 2018). Pipettes pulled from borosilicate glass had a resistance of 4-6 $\mathrm{M} \Omega$ when filled with the intracellular solution: $120 \mathrm{~mm}$ potassium gluconate, $10 \mathrm{mM} \mathrm{KCl}$, 10 mм HEPES, 0.2 mм EGTA, 4.5 mм MgATP, 0.3 mм NaGTP, 14 mм sodium phosphocreatine, $0.1 \%-0.2 \%$ neurobiotin, $\mathrm{pH}$ adjusted to $7.2-7.4$ with $\mathrm{KOH}$. Recordings were obtained using Multiclamp 700B amplifiers (Molecular Devices). Signals were low-pass filtered at $6 \mathrm{kHz}$ and digitized at $20 \mathrm{kHz}$ using DigiData 1200 acquisition interfaces (Molecular Devices). Data acquisition and analysis were performed using Clampex 10.2 and ClampFit 10.2 software (Molecular Devices). Only recordings with a stable series resistance of $<20 \mathrm{M} \Omega$ were used for analysis. In current clamp, series resistance and pipette capacitance were canceled using bridge and capacitance neutralization. Cells included in this study had 
resting membrane potential between -60 and $-80 \mathrm{mV}$. EPSPs were elicited in the pyramidal neurons by stimulating the ChR2-expressing vHP terminals with $447 \mathrm{~nm}$ light-emitting diode (Tolket) through a water immersion $40 \times$ microscope objective $(15 \mathrm{~mW}$ in the focal plane of the objective). Blue light-evoked responses were considered monosynaptic when exhibiting $\mathrm{a} \leq 4 \mathrm{~ms}$ latency from light onset (Bogart and O'Donnell, 2018) and a jitter $<1.2 \mathrm{~ms}$ (measured as SD of 30 trials) (Marek et al., 2018). After recordings, the slices were quickly fixed with PFA (4\% in PBS), and recorded neurons were imaged using fluorescence-labeled streptavidin (streptavidin-Cy3, Invitrogen). The area of the vHP transduced by the AAV virus was estimated by measuring the area of EYFP fluorescence in the CA1 region of the coronal plane with the largest transduction of EYFP, and the septal-temporal span of infection was estimated by counting the number of continuous coronal planes containing CA1 labeled with EYFP.

\section{Postmortem histology}

At the end of each recording session, mice received a lethal dose of urethane and were transcardially perfused with $10 \mathrm{ml}$ cold saline solution followed by $20 \mathrm{ml}$ of buffered PFA (4\% in PBS). Brains were removed, immersed overnight in the same fixative at $4^{\circ} \mathrm{C}$, and stored in $0.1 \mathrm{M} \mathrm{PB}$ containing $15 \%$ sucrose at $4^{\circ} \mathrm{C}$ for $24-72 \mathrm{~h}$. Coronal brain sections were serially cut with a microtome with a freezing stage $(50 \mu \mathrm{m})$ for histologic reconstructions. Location of the concentric bipolar electrodes was assessed by visual examination of the mechanical tissue damage in the coronal sections stained with Safranin O using a transmitted light microscope at low magnification. In some experiments, tetrodes were immersed in a red fluorescent dye $\left(1,1^{\prime}\right.$ dioctadecyl-3,3,3' $3^{\prime}$-tetramethylindocarbocyanine perchlorate, $100 \mathrm{mg} /$ $\mathrm{ml}$ in acetone; DiI, Invitrogen) and air-dried for $30 \mathrm{~min}$ before use. This allowed detecting the fluorescent material deposited in the tissue with an epifluorescence microscope. In all cases, photomicrographs were obtained from consecutive sections of $\mathrm{mPFC}$ and hippocampus to confirm placement of recording and stimulation sites. Only experiments were correct electrode placement was verified are included in the analysis.

\section{Experimental design and statistical analyses}

Data were analyzed using actorial model ANOVAs. When significant differences were detected, ANOVAs were followed by a Newman-Keuls post hoc test for multiple comparisons. The distribution of discrete variables between groups was compared using the $\chi^{2}$ test. Individual stimulus-response curves were parameterized by fitting a sigmoidal function (Boltzmann, by least squared fitting). Maximal amplitude and slope obtained from individual sigmoidal function were analyzed using ANOVAs. Data are mean \pm SEM, except when indicated otherwise. Two-tailed parametric statistics were used in all cases, and the threshold for significance was set at $p=0.05$. The $p$ values are indicated along with statistical parameters, such as number of cells and animals used in each experiment in Results and in the figure legends. No animals or neurons that fulfill our quality single-unit inclusion criteria were excluded from the analysis. Post hoc power analysis was performed for all statistical comparisons, although its value has been questioned in retrospective calculations (Hoenig and Heisey, 2001). Statistical analyses were conducted using Statistica 7.0 (StatSoft), and fitting curves were obtained with Prism 4.0 (GraphPad Software).

\section{Results}

Our previous studies have shown that early-postnatal ablation (between PD7 and PD21) of Grin1 in cortical and hippocampal $\mathrm{PV}^{+}$interneurons leads to SZ-related phenotypes once mutant mice reach young adulthood ( $>8$ weeks old) (Belforte et al., 2010; Jiang et al., 2013). In order to elucidate the pathophysiological changes underlying altered adult behavior, we assessed the impact of cortical interneuron early postnatal NMDAR manipulation on neural activity before and after adolescence. In vivo electrophysiological activity was recorded in $\mathrm{mPFC}$ of
A

PYRAMIDAL CELLS
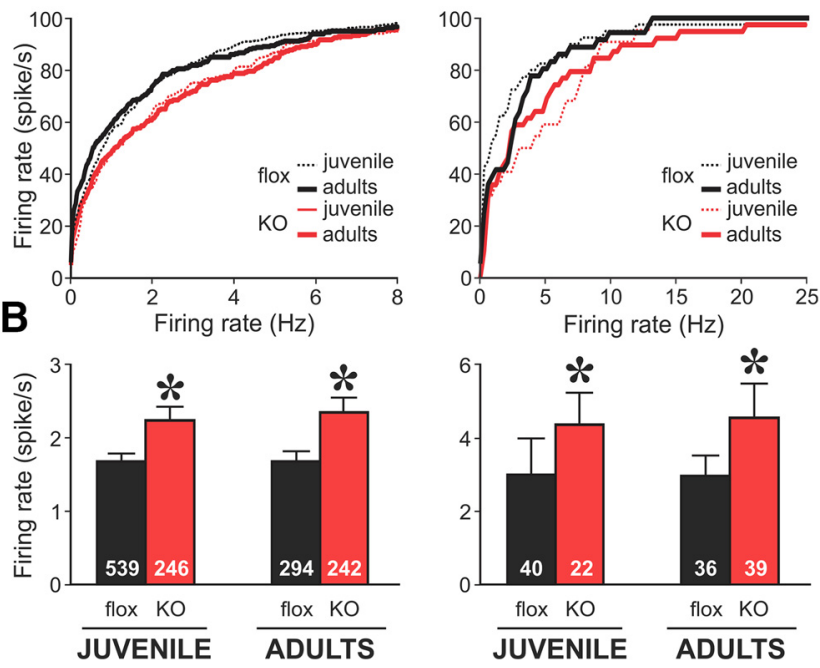

Figure 2. 'Increased pyramidal and interneuron firing rate in juvenile and adult NMDARKO mice. $A$, Cumulative frequency distribution of pyramidal (left) and interneuron (right) firing rate of control and NMDAR-KO mice quantified during desynchronization periods. The curves for juvenile and adult mice are shifted to the right in the NMDAR-KO mice. $\boldsymbol{B}$, Overall increase in PFC pyramidal and interneuron mean firing rate in the NMDAR-KO mice (twoway ANOVAs: genotype factor, pyramidal). ${ }^{*} p<0.0001$ versus control, interneurons. ${ }^{*} p=$ 0.01 versus control. Data are mean \pm SEM. Number of cells are indicated inside columns from 11 juvenile control, 7 juvenile KO, 6 adult control, and 6 adult $\mathrm{KO}$ mice.

anesthetized juvenile (PD28-PD32) and adult mutant and control mice (Fig. 1A). The use of general anesthesia allowed us to minimize the impact of peripheral inputs that may vary across developmental stages (Cheetham and Fox, 2010; Caballero et al., 2014a) and differences due to the behavioral differences expected for mutants and controls.

We recorded a total of 909 and 549 well-isolated units from control and mutant mice, respectively, along the entire mPFC (Fig. 1A). Isolated units were offline classified into two subclasses (putative pyramidal cells and interneurons) based on waveform and firing characteristics (Fig. $1 B$ ). Putative pyramidal neurons were defined as cells with relatively broad spike waveforms and with negative curvilinear shapes in the afterhyperpolarization phase. Based on their relatively narrow waveform, $9.4 \%$ of all recorded cells (137 of 1458 units) were considered putative interneurons, and most likely correspond to fast-spiking interneurons (percentage of interneurons does not differ across groups, control: $9.1 \%$ vs KO: $12.5 \%)$. Visual inspection of the scatter plots of mean spike waveform durations did not show any evident waveform differences between mutant and controls; Fig. $1 B$ ). We restricted our analysis to desynchronized cortical LFP periods given that, during those segments, global activity resembles that observed in awake animals, it is not purely governed by local inputs, and a prominent theta oscillation is present in the vHP (Fig. 1C).

Given that one of the more canonical functions of GABAergic interneurons (in particular, fast-spiking ones) is to regulate the activity of the cortical network (Curtis et al., 1970; Packer and Yuste, 2011), we first assessed the impact of Grin1 ablation on firing rate of pyramidal cells and interneurons. As expected from our previous recordings in somatosensory cortex (Belforte et al., 2010), we found that Grin1 ablation in adults results in an increase in the mean firing rate of pyramidal cells (Fig. $2 A$; two-way ANOVA, significant genotype factor, $F_{(1,1317)}=$ 
A

Theta locked Theta non-locked pyramidal pyramidal

B
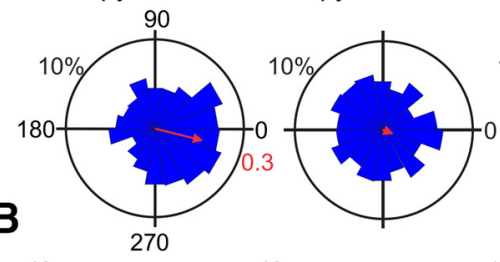

Theta locked interneuron

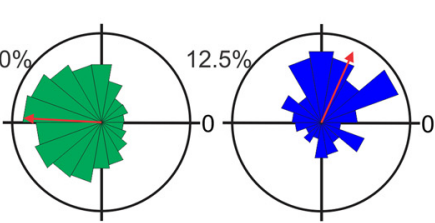

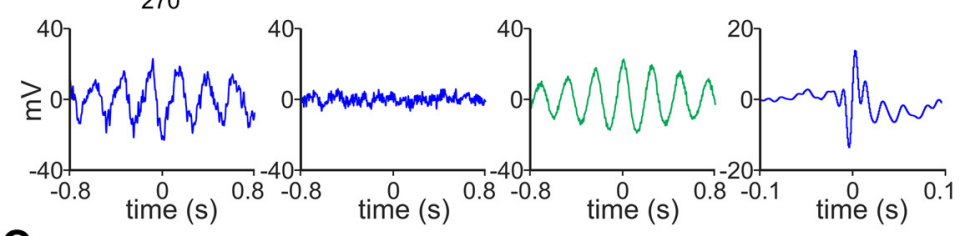

C

PYRAMIDAL CELLS
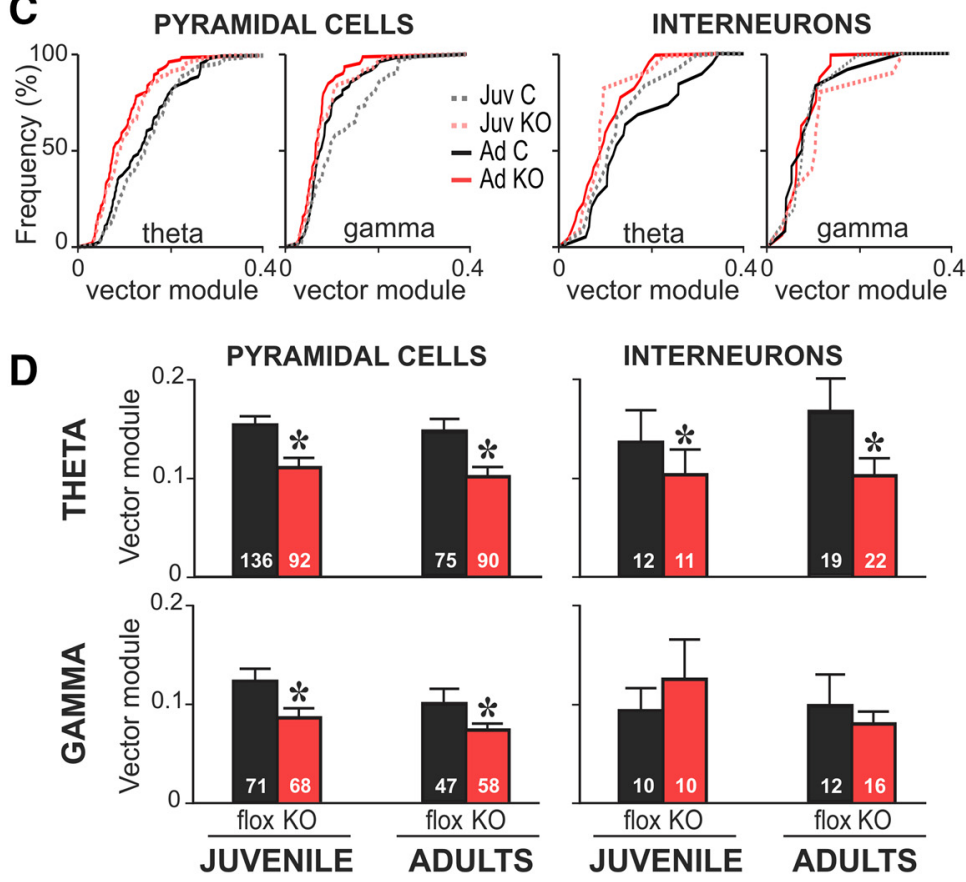

Figure 3. Phase-locking of mPFC neurons to local and distal rhythms is decreased in juvenile and adult NMDAR-KO mice. $\boldsymbol{A}$, Representative examples of circular phase plots representing the occurrence of spike discharges (radial axis) as a function of LFP oscillation phase (angular axis) for 4 representative mPFC cells. Bin size $20^{\circ}$. Examples include pyramidal cells (blue) and interneurons (green) locked and not phase-locked to hippocampal theta or cortical $\gamma$ rhythms. Red arrows indicate the resultant vector of the circular phase distribution. Red numbers indicate the radial axes for vector module. Left, First example presents a pyramidal cell phase-locked to hippocampal theta rhythm as evidenced by a nonuniform circular distribution (Rayleigh test, $p<0.001$ ). $\boldsymbol{B}$, Spike-triggered LFP average for each of the neurons plotted in $A$. STAs from phase-locked units reproduce the underlying oscillation. $C$, Cumulative frequency distributions of phaselocking strength to VHP theta and cortical $\gamma$ oscillations for all phase-locked (Rayleigh, $p<0.05$ ) pyramidal and interneurons from juvenile (Juv) and adult ( $\mathrm{Ad}$ ) control $(\boldsymbol{C})$ and NMDAR-KO mice (KO). $\boldsymbol{D}$, Phase-locking strength is represented as the average vector module for all phase-locked neurons (Rayleigh, $p<0.05$ ) for each experimental group. Irrespective of age, neurons from $\mathrm{KO}$ mice show a decreased phase-locking to hippocampal theta rhythm (two-way ANOVA, genotype factor pyramidal: ${ }^{*} p<0.0001$; interneurons: ${ }^{*} p=0.018$ ). Pyramidal cells from KO mice displayed significant lower locking to cortical $\gamma$ LFP oscillation at both ages (two-way ANOVA, pyramidal: genotype factor, ${ }^{*} p<0.0001$ ). No changes were observed for interneurons in relation to $\gamma$ rhythm (two-way ANOVA, genotype factor: $p=0.67)$. No significant interactions between age and genotype factors were detected by two-way ANOVAs in the statistical analysis. Data are mean \pm SEM of $n$ cells indicated in the graph bars from 11 juvenile control, 7 juvenile KO, 6 adult control, and 6 adult $\mathrm{KO}$ mice.

$15.8 p<0.0001$ vs control, observed power: 0.978 , nonsignificant interaction, $\left.F_{(1,1317)}=0.12 p=0.73\right)$. Interestingly, juvenile mutant mice also present a significant increase in pyramidal neurons firing rate, suggesting that this electrophysiological phenotype is not itself responsible for the SZ-like phenotype exhibited by adults. To fully characterize the physiological changes, we analyzed the mean firing rate of putative interneurons. Surprisingly, Grin1 ablation results in a significant increase in the mean firing rate of interneurons regardless of the developmental stage analyzed (Fig. $2 B$; significant genotype factor $F_{(1,133)}=6.82, p=$ 0.010 vs control, observed power: 0.73 , nonsignificant interaction $F_{(1,133)}=0.24$ $p=0.63$, observed power: 0.07$)$. Thus, it appears that the increase in pyramidal neuron activity observed in mutants is counterbalanced by a higher inhibitory drive. Indeed, the pyramidal/interneuron firing ratio is similar in control and mutant mice, regardless of the age analyzed. Together, this suggests that cortical network of Grin1 mutants is balanced around a different set point for mean firing rate but operates with a similar dynamic range at population level.

GABAergic interneurons not only regulate firing rate, but also spike timing and synchronization (Pouille and Scanziani, 2001; Wehr and Zador, 2003; Hasenstaub et al., 2005), allowing encoding of information by locking the relative timing of spikes in the neuronal population with respect to an ongoing brain oscillation (Somogyi and Klausberger, 2005; Haider and McCormick, 2009; Uhlhaas and Singer, 2010; Buzsáki and Wang, 2012). In addition, altered neuronal synchronization with cortical oscillations has been proposed as an underlying pathophysiological mechanism involved in cognitive and perceptual deficits reported in SZ patients (Spencer et al., 2003; Cho et al., 2006). Therefore, we next asked whether NMDAR hypofunction in cortical and hippocampal $\mathrm{PV}^{+}$interneurons may disrupt spike synchronization to relevant oscillatory brain activity. We focused our study to a locally generated rhythm (cortical $\gamma$ oscillation) (Sirota et al., 2008) and another one emerging distally to the mPFC (hippocampal theta oscillations). Previous studies have shown that $\mathrm{PV}^{+}$cells are involved in control and generation of cortical $\gamma$ oscillations (Buzsáki and Wang, 2012). Fast-spiking interneurons have also been associated with feedforward inhibition of distal inputs, including monosynaptic input arriving from the vHP (Gigg et al., 1994; Gabbott et al., 2002; Dégenètais et al., 2003). For well-isolated mPFC units, we quantified the degree of synchronization to the theta and $\gamma$ oscillations by plotting the rate of occurrence of spikes as function of the oscillations' phase (Fig. 3A). Thus, if a neuron fires preferentially at the trough of a given oscillation, this will produce an asymmetric circular distribution with a peak at $0^{\circ}$. This asymmetry can be summarized with a vector whose angle and length are the phase and strength of entrainment, respectively (Fig. $3 A$ ). Units were considered entrained to a given frequency band if the circular phase distribution significantly 

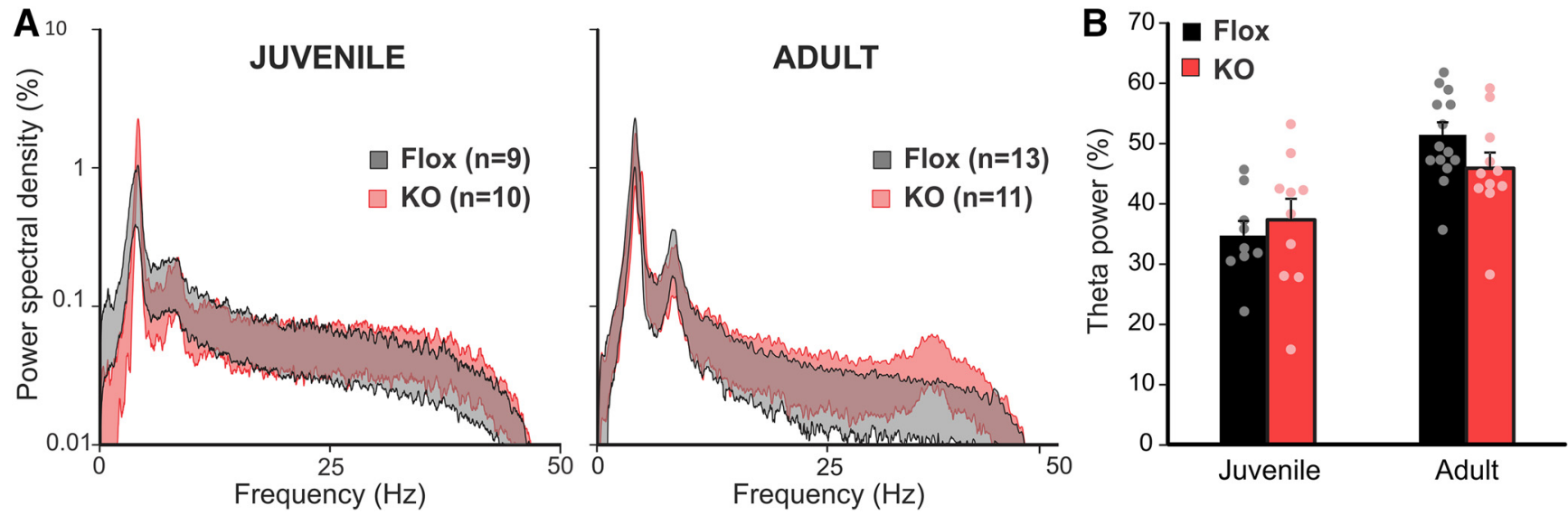

Figure 4. $\quad$ vHP theta oscillation is not affected in NMDAR-KO mice. $\boldsymbol{A}$, Power spectral density analysis of LFP signals recorded from vHP in juvenile and adult control and NMDAR-KO mice during desynchronized states expressed as a percentage of the total spectral power. Data are mean \pm SEM of $n$ recorded animals. $\boldsymbol{B}$, Power densities were calculated per band for theta oscillation (3-7 Hz). No statistically significant differences were observed across genotypes. Data are mean \pm SEM. Each dot represents 1 individual animal.

deviated from uniformity ( $p<0.05$ Rayleigh test). As reported previously (Sirota et al., 2008), mPFC interneurons and pyramidal cells can be both entrained (i.e., phase-locked) to vHP theta and cortical $\gamma$ oscillations (Fig. 3A). This neuronal entrainment can also be visualized by performing an STA of the corresponding LFPs, demonstrating phase-locking to physiological relevant oscillations (Fig. 3B). Interestingly, our results show a significant reduction in the magnitude of $\mathrm{mPFC}$ pyramidal and interneurons phase-locking to vHP theta oscillation, both in juvenile and adults mutant mice compared with age-matched controls (Fig. $3 C, D$; two-way ANOVA, pyramidal: genotype factor $F_{(1,389)}=$ 31.7, $p<0.0001$, observed power: 0.99; interneurons: genotype factor $F_{(1,60)}=5.90, p=0.018$, observed power: 0.67 , no significant interactions). The reduction is similar in both developmental stages, and no significant interaction was observed. However, the proportion of significantly entrained pyramidal units in juvenile mutants is marginally, but significantly, increased compared with control mice (control 37.2\%, 136 of 365 vs KO 47.7\%, 92 of 193 units; $\chi^{2}$ test $=5.66 p=0.017$, observed power: 0.67 ). Therefore, in mutants, more neurons in $\mathrm{MPFC}$ are synchronized to the vHP theta rhythm, but their entrainment is less reliable. Since NMDAR ablation may impact on hippocampal activity of KO mice, we analyzed the oscillatory components in vHP LFP signals recorded from control and KO mice using FFT analysis. Power spectral analysis did not show differences between experimental groups (Fig. 4A). Furthermore, there are no significant differences across genotypes at either developmental stage in the vHP theta band power (Fig. $4 B$; two-way ANOVA, genotype factor, $F_{(1,39)}=0.39 p=0.58$, observed power: 0.084$)$ supporting our interpretation that decreased vHP theta entrainment of $\mathrm{mPFC}$ neurons in $\mathrm{KO}$ mice does not arise from a deficit in the vHP itself but rather from the synchronization of mPFC units to the incoming signal. These results suggest that a correcting mechanism might occur at the population level in an attempt to restore global network synchrony at the juvenile stage. In adult mutant mice, this compensation is lost since no significant differences were observed in the percentage of entrained units (control $43.1 \%, 75$ of 174 vs $\mathrm{KO} 48.4 \%, 90$ of 186 units; $\chi^{2}$ test $=1.01$ $p=0.31$, observed power: 0.17 ), probably leading to an altered processing of vHP inputs to $\mathrm{mPFC}$. Indeed, this scenario is not specific to distal inputs since $\mathrm{mPFC}$ pyramidal cells show a similar altered profile in relation to locally generated $\gamma$ oscillations. Where entrainment magnitude of pyramidal cells to $\gamma$ is significantly lower in mutant mice of both ages (Fig. $3 C$, bottom; two-way ANOVA, pyramidal: genotype factor, $F_{(1,240)}=16.3$, $p<0.0001$, observed power: 0.98$)$ and the number of significantly phase-locked units is increased only in juvenile mice (juvenile: control $19.5 \%$ vs $\mathrm{KO} 35.2 \%, \chi^{2}$ test $=16.8 p<0.0001$, observed power: 0.56 ; adults: control $27.0 \%$ vs $\mathrm{KO} 31.2 \%, \chi^{2}$ test $=0.76 p=0.38$, observed power: 0.067 ). Analysis of interneurons' entrainment shows an age-independent deficit for theta rhythm but a normal $\gamma$ synchrony (two-way ANOVA, genotype factor, $F_{(1,44)}=0.18, p=0.67$, observed power: 0.07$)$. In summary, early postnatal ablation of Grin 1 clearly alters $\mathrm{mPFC}$ neuronal synchronization to local and distally generated inputs, but this altered processing is already present in juvenile asymptomatic mice.

Interneurons, and $\mathrm{PV}^{+}$in particular, are not only associated with control of firing rate and spike timing, but they are also involved in controlling the response of a network to incoming inputs. It has been proposed that $\mathrm{PV}^{+}$cells can modulate cortical gain (Atallah et al., 2012; Lee et al., 2012) and a networks' dynamic range (Pouille et al., 2009; Atallah et al., 2012). In order to probe whether $\mathrm{PV}^{+}$NMDAR hypofunction leads to age-dependent changes in cortical responsiveness to excitatory inputs, we measured $\mathrm{mPFC}$-evoked responses to vHP stimulation before and after adolescence in mutants and control mice (Fig. 5A). Stimulation of vHP evoked a well-described, short-latency, response in $\mathrm{mPFC}$ LFP (Fig. $5 B$, inset). The stimulus-response curves for juvenile mice did not show any significant difference between genotypes either for threshold or maximal response amplitude (Fig. $5 B$, left; genotype factor: $F_{(1,490)}=0.50, p=0.49$, genotype $\times$ intensity interaction: $F_{(14,490)}=0.84, p=0.62$, observed power: 0.54$)$. Remarkably, the stimulus-response curve for adult mutant mice exhibited a marked reduction in its maximal evoked response compared with control age-matched mice (Fig. $5 B$, right; two-way repeated-measures ANOVA, genotype $\times$ intensity interaction: $F_{(13,325)}=3.12 p<0.001$, observed power: $0.99)$. We detected a significant interaction between genotype and developmental stage for maximal stimulation evoked response (Fig. $5 C$; two-way ANOVA, genotype $\times$ age interaction $F_{(1,60)}=5.44 p<0.05$, observed power: 0.63$)$. Also, adult control mice showed a significant reduction in the maximal evoked 
A
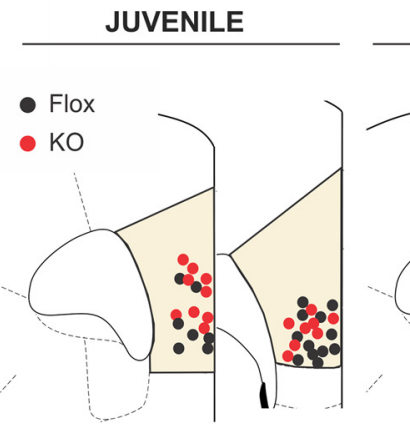

ADULTS

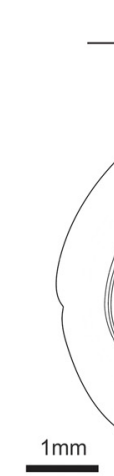

B
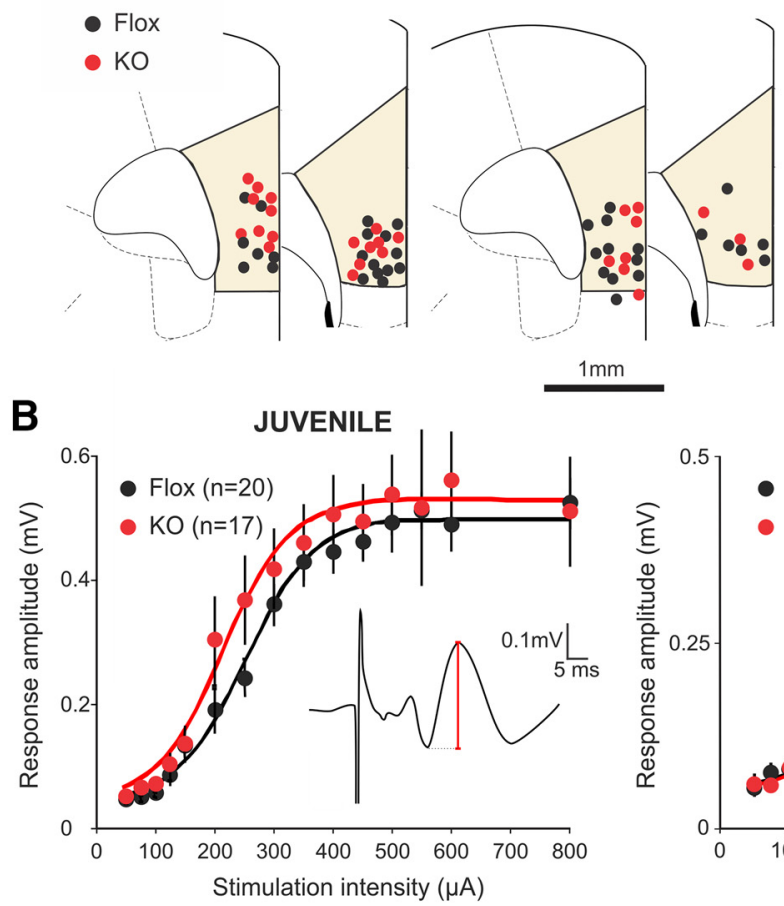

$1 \mathrm{~mm}$
JUVENILE ADULTS ADULTS

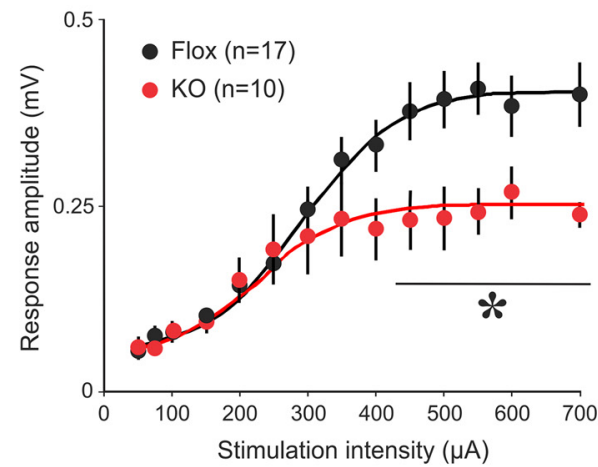

C

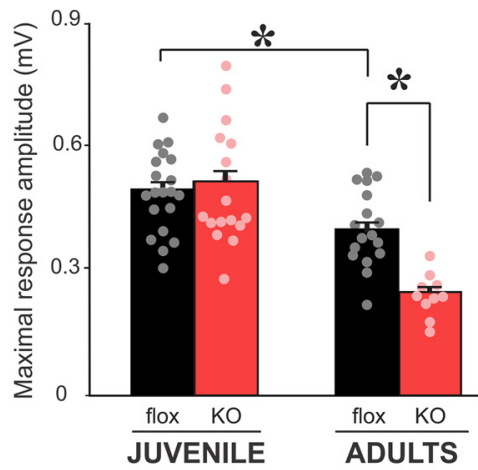

Figure 5. Only adult NMDAR-KO mice show a reduced mPFC-evoked response to vHP stimulation. $\boldsymbol{A}$, Schematic of coronal sections showing recording (mPFC; left) and stimulation (vHP; right) electrode positioning for juvenile and adult control and KO mice. $\boldsymbol{B}$, Stimulation of vHP evoked a short-latency response in mPFC LFP (inset; red line indicates amplitude of the evoked response). mPFC stimulus-response curves evoked by stimulation of vHP show a significant decrease in response amplitude in adult, but not juvenile, KO mice compared with age-matched control mice (two-way repeated-measures ANOVAs). Adult: genotype $\times$ intensity interaction, $p<0.001$. ${ }^{*} p<0.05$ (Newman-Keuls post hoc test vs control at same stimulation intensity). Juvenile: genotype factor, $p=0.49$; genotype $\times$ intensity interaction, $p=0.62$. Each point represents mean \pm SEM. Number of mice is indicated. Trend line indicates the best fit sigmoidal curves to population data. C, Maximum evoked mPFC response to vHP stimulation. Adult KO mice show a decreased maximal evoked response compared with age-matched control mice, although no differences were observed between control and KO juvenile mice (two-way ANOVA, genotype $\times$ age interaction). ${ }^{*} p<0.05$ (Newman-Keuls post hoc test). Error bar indicates mean \pm SEM. Each dot represents 1 individual animal.

response compared with juvenile control mice, probably reflecting a normal refinement in the functional connectivity between vHP and mPFC during adolescence. The abnormally increased age-dependent functional disconnection observed in mutant mice resembles connectivity deficits already reported for this pathway in SZ patients (Ford et al., 2002; Lawrie et al., 2002; Meyer-Lindenberg et al., 2005; Worbe et al., 2015).

Since the result obtained for Grin1 mutants can be interpreted as an overreduction in the normal refinement that takes place during adolescence and GABAergic interneurons have been implicated in control of network plasticity (Hensch et al., 1998; Donato et al., 2013; Kuhlman et al., 2013), we wondered whether this loss in functional connectivity was accompanied by an altered in vivo plasticity. Thus, we evaluated the vHP-mPFC pathway susceptibility to suffer LTD in vivo. LFS vHP induced an $\sim 60 \%$ reduction of the $\mathrm{mPFC}$ response that lasted up to $1 \mathrm{~h}$ after LFS stimulation ends (Fig. 6A). While LFS-LTD is indistinguishable in mutants and controls before adolescence (Fig. 6B, top; two-way repeated-measures ANOVA: genotype factor, $F_{(1,384)}=0.18 p=0.68$, genotype $\times$ time interaction, $F_{(24,384)}=$ $1.09 p=0.35$, observed power: 0.85 ), adult mutants presented a higher susceptibility to vHP-LFS, showing a greater reduction of the basal-evoked response compared with controls (Fig. 6B, bottom; two-way repeated-measures ANOVA: genotype $\times$ time interaction, $F_{(24,240)}=1.94 p<0.01$, observed power: 0.99$)$. We subjected all experimental groups to three additional LTD-LFS to determine the LTD saturation level. In juvenile mutants, LTD saturation was similar to control group (Fig. 6A-C). In adults, although LTD was saturable in both genotypes, a small but significant difference was observed regarding saturation magnitude, with mutants exhibiting a more pronounced maximal depression after LTD saturation was reached (Fig. 6C). Combined statistical analysis of juvenile and adults groups showed a significant interaction between genotype and age (three-way repeated-measures ANOVA: genotype $\times$ age interaction, $F_{(1,78)}=4.93, p=0.035$, observed power: 0.57$)$. This result provides a putative mechanism to explain the reduced functional connectivity between vHP and mPFC that emerges after adolescence in Grin1-ablated mice. To explore whether the observed increased in LTD in adult mutants reflects a general propensity toward higher plasticity, we evaluated, in an independent group of animals, the status of the vHP-mPFC pathway response to HFS-LTP. As reported previously, HFS of the vHP induced a marked LTP response in MPFC (Fig. 7A). Interestingly, HFS-LTP in juvenile mutants is significantly facilitated compared with age-matched controls (Fig. $7 B$; two-way repeated-measures ANOVA: genotype $\times$ time interaction, $F_{(24,384)}=4.70, p<0.0001$, observed power: 0.99). However, adult mutant mice displayed nonsignificant differences in LTP induction and saturation levels (Fig. 7B; two-way repeated-measures ANOVA: genotype factor, $F_{(1,312)}=0.07$ $p=0.79$, genotype $\times$ time interaction, $F_{(24,312)}=0.95 p=0.54$, observed power: 0.77$)$. Combined analysis across ages evidenced an enhanced HFS-LTP in juvenile mutants that is normalized at adulthood (Fig. 7C), indicating that the higher sensibility to LFS- 
A
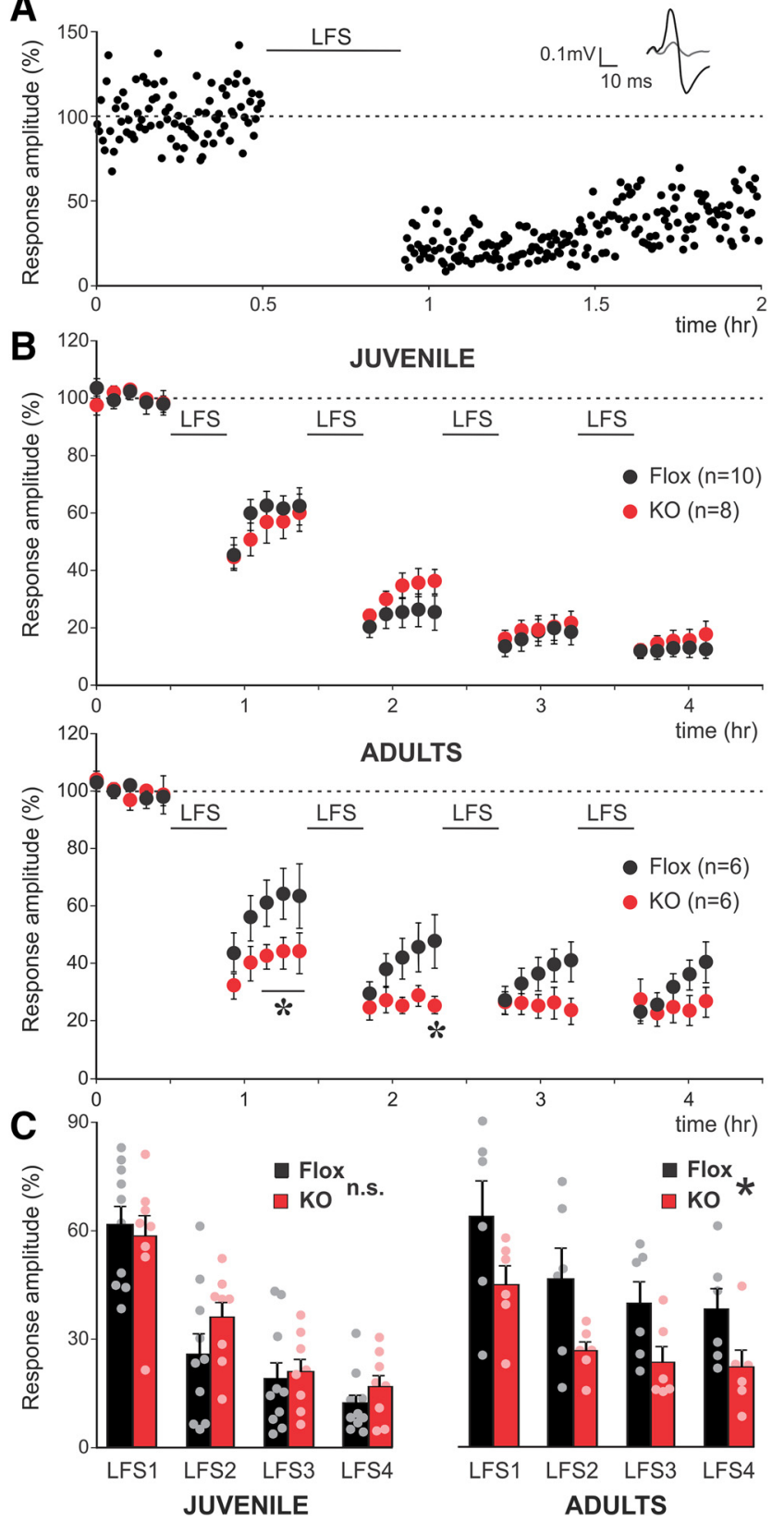

Figure 6. Adult KO mice present a higher susceptibility to VHP-LFS induced LTD. A, Representative experiment illustrating time course of mPFC-evoked response before and after LFS stimulation of vHP. Each dot represents amplitude of the response evoked by a test pulse delivered to vHP. Inset, Traces of mPFC-evoked responses before (black) and after (gray) LFS stimulation. The LFS protocol induces a long-lasting depression of the evoked response. Response amplitudes were normalized to mean baseline amplitude. $\boldsymbol{B}, \mathrm{mPFC}$ response amplitude recorded from animals subjected to successive LFS protocols. Juvenile control and KO mice showed similar levels of LTD after application of successive LFS protocols (two-way repeated-measures ANOVA: genotype factor, $p=0.68$; genotype $\times$ time interaction, $p=0.35$ ). Adult $K 0$ mice presented greater levels of induced LTD than control mice (two-way repeated-measures ANOVA: genotype $\times$ time interaction, $p<0.01$ ). ${ }^{*} p<0.05$ (Newman-Keuls post hoc test). Each point represents mean \pm SEM of $n$ animals. $C$, The mPFC-evoked response is smaller after each of the LFS protocols in adult, but not in juvenile, NMDAR-KO. Statistical analysis combining genotype, age, and LFS repetition was conducted by three-way repeated-measures ANOVA (genotype $x$ age interaction, $p=0.035$ ). ${ }^{*} p<0.05$ (Newman-Keuls post hoc test vs adult control). Error bar indicates mean \pm SEM. Each dot represents the response measured in 1 individual animal. n.s., not significant.
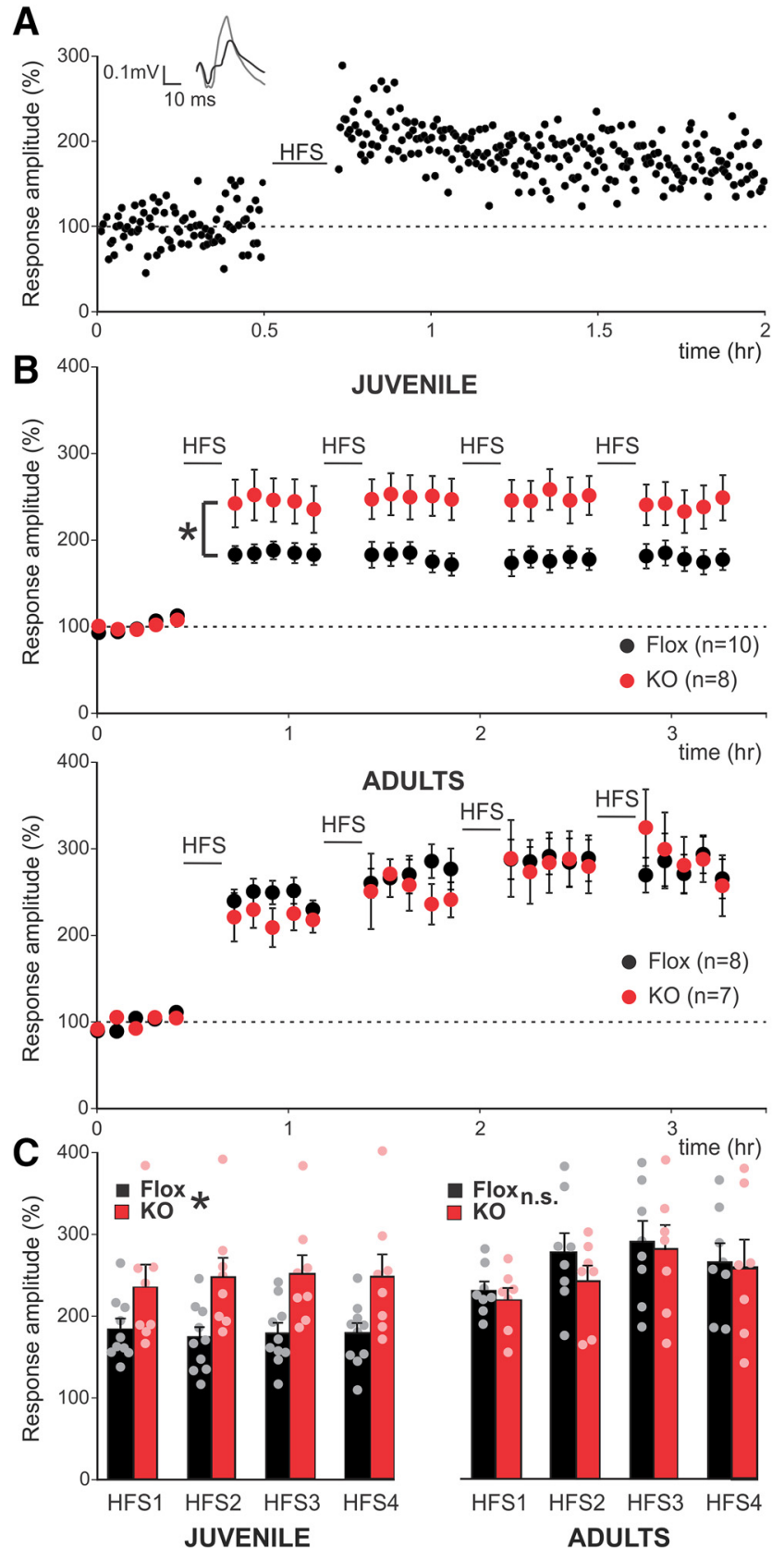

Figure 7. Adult KO mice exhibit normal susceptibility to vHP-HFS induced LTP. A, Representative experiment illustrating time course of mPFC-evoked response before and after HFS stimulation of VHP. Each dot represents amplitude of the response evoked by a test pulse delivered to vHP. Inset, Traces of mPFC-evoked responses before (black) and after (gray) HFS stimulation. The LTP protocol induces a long-lasting potentiation of the evoked response. Response amplitudes were normalized to mean baseline amplitude. $\boldsymbol{B}, \mathrm{mPFC}$ response amplitude recorded from animals subjected to successive HFS protocols. A higher susceptibility to HFS in juvenile KO mice results in a significant increase in the evoked response compared with control mice after application of successive HFS pulse trains (twoway repeated-measures ANOVA: genotype $\times$ time interaction, $p<0.0001$ ). ${ }^{*} p<0.05$ (Newman-Keuls post hoc test vs adult control). Each point represents mean \pm SEM of $n$ animals. In contrast, adult control and KO mice showed no significant differences in LTP levels after application of successive HFS protocols (two-way repeated-measures ANOVA: genotype factor, $p=0.79$; genotype $\times$ time interaction, $p=0.54$ ). $C$, The mPFC-evoked response is bigger after each of the HFS protocols in juvenile, but not in adult, NMDAR-KO. Statistical analysis combining genotype, age, and LFS repetition was conducted by three-way repeatedmeasures ANOVA (genotype $\times$ age interaction, $F_{(1,87)}=4.80, p=0.036$; observed power: $0.56) .{ }^{*} p<0.05$ (Newman-Keuls post hoc vs juvenile control). Error bar indicates mean \pm SEM. Each dot represents the response measured in 1 individual animal. n.S., not significant. 
LTD in adult mutants is not the result of a general alteration of plasticity mechanisms in the mPFC. Accordingly, LTD and LTP developmental profiles in mutants follow opposite trajectories, supporting the view that a developmental shift from potentiation to depotentiation occurs in mutant mice along adolescence.

It is well established that $\mathrm{PV}^{+}$interneurons regulate the functional impact of excitatory inputs, including vHP ones (Tierney et al., 2004), on PFC pyramidal neurons by feedforward and feedback inhibition. Thus, the vHP-mPFC hypoconnectivity and the increased LTD of the hippocampal inputs to the mPFC in the adult KO may arise from a deregulated local GABAergic processing instead of a decrease in the direct monosynaptic input to $\mathrm{mPFC}$ pyramidal cells. Thus, to evaluate whether the decreased vHP-mPFC functional connectivity displayed by adult $\mathrm{KO}$ mice is independent of the local GABAergic circuit, we used an optogenetic approach to selectively stimulate vHP terminals on $\mathrm{mPFC}$ in brain slices under GABAergic blockade. Blue light illumination of ChR2-expressing vHP terminals (Fig. 8A,B) reliably evoked EPSPs in $\mathrm{mPFC}$ layer three pyramidal neurons in $e x$ vivo slices from control and mutant adult mice with no failures, low response jitter, and latency (Fig. 1C, top) consistent with direct monosynaptic connections (Bogart and O'Donnell, 2018; Marek et al., 2018). No differences were observed between control and mutant mice regarding EPSP response jitter (Fig. 1C, bottom; Student's $t$ test, $t_{(15)}=0.66, p=0.51$ ), onset latency (controls: $3.21 \pm 0.24 \mathrm{~ms}$, mutants: $2.87 \pm 0.21 \mathrm{~ms}$; Student's $t$ test, $t_{(15)}=0.51, p=0.62$ ), and peak latency (controls: $7.74 \pm 0.70 \mathrm{~ms}$, mutants: $7.65 \pm 0.23 \mathrm{~ms}$; Student's $t$ test, $\left.t_{(15)}=0.03 p=0.97\right)$. However, reinforcing and extending our proposal of a vHP$\mathrm{mPFC}$ functional hypoconnectivity in adult mutant mice, we found a significant reduction in the amplitude of the monosynaptic EPSPs evoked by light stimulation of vHP terminals in pyramidal neurons that is independent of GABAergic activity in the mutants (Fig. $8 D$; Student's $t$ test, $t_{(16)}=2.50, p=0.023$, observed power 0.96). Importantly, the extent of transduction of CA1 in the vHP was similar in the experimental groups both at the injection site (controls: $1.102 \pm 0.077 \mathrm{~mm}^{2}$, mutants: $1.136 \pm 0.113 \mathrm{~mm}^{2}$; Student's $t$ test, $t_{(10)}=0.48, p=0.64$ ) and in the septal-temporal spread (controls: $1.67 \pm 0.09 \mathrm{~mm}$, mutants: $1.62 \pm 0.15 \mathrm{~mm}$; Student's $t$ test, $t_{(10)}=0.31, p=0.76$ ).

\section{Discussion}

Here we assessed the pathophysiological resultant of an early postnatal ablation of the NMDAR selectively in cortical and hippocampal $\mathrm{PV}^{+}$interneurons as an animal model of SZ. We found that cortical hyperactivity and uncoupled firing to oscillations occur in mutant mice before adolescence, and can be the direct consequence of the interneuron dysfunction. Instead, mPFC functional hypoconnection with vHP occurs in mutants after adolescence. Our results support the idea that abnormal plasticity during the remodeling period in adolescence may be a key factor for the emergence of the hypoconnectivity observed in adult mutant mice.

A substantial number of pathophysiological mechanisms subjacent to SZ symptoms have been proposed, including dopaminergic misbalance, altered cortical interneuron function, and deficits in long-range functional connectivity. Given the multiplicity of symptoms and the diversity of clinical manifestations exhibited by SZ patients, it is likely that several pathophysiological alterations contribute simultaneously to the final clinical outcome. Even more, pathophysiological changes are probably interrelated, making difficult to determine which ones are causal
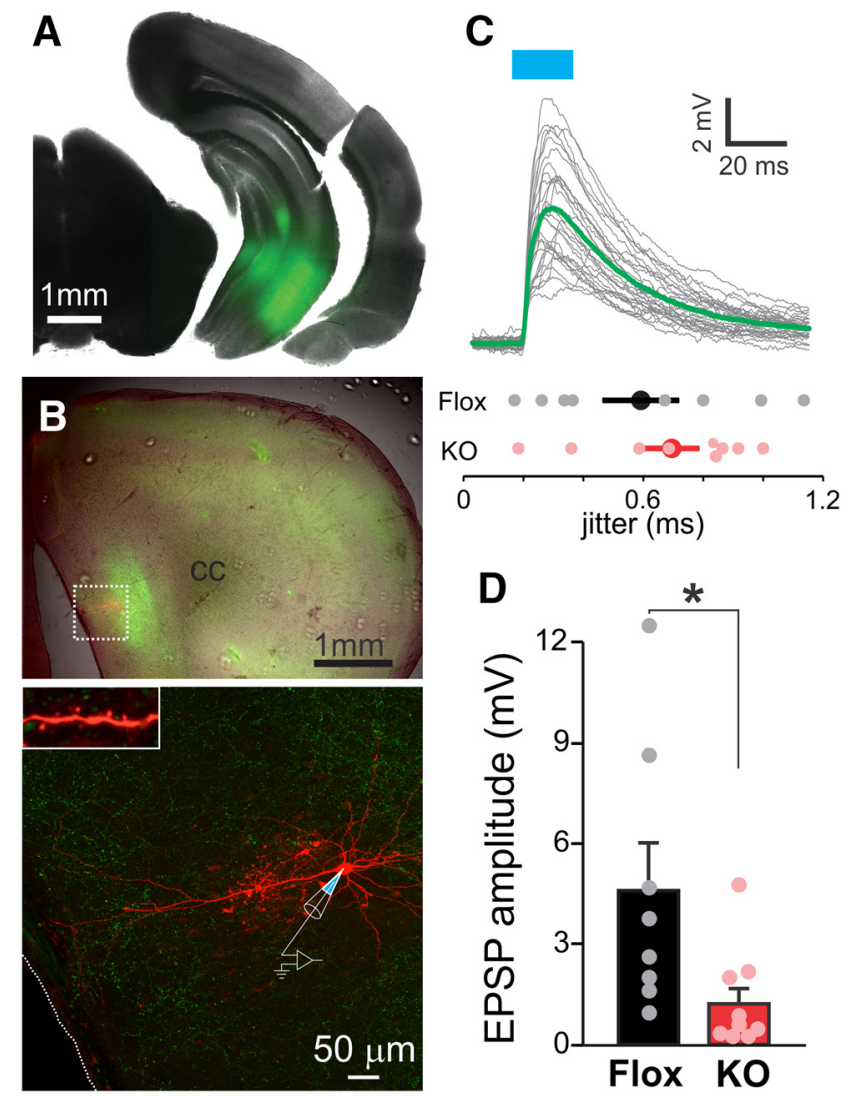

Figure 8. KO mice show decreased amplitude of monosynaptic EPSPs in mPFC pyramidal neurons evoked by optogenetic stimulation of vHP terminals. $\boldsymbol{A}$, vHP microinjection of AAV2/ CamKII-hChR2(H134R)-EYFP results in selective EYFP fluorescence (green) expression in transduced $\mathrm{vHP}$ region. Low-magnification photomicrograph shows a representative example of the vHP targeted area. $\boldsymbol{B}$, Top, Low-power photomicrograph of a representative brain slice (300- $\mu \mathrm{m}$-thick) used for patch-clamp recordings in mPFC after streptavidin-Cy3 staining for visualization of the neurobiotin-filled recorded neuron. Dense innervation of vHP terminals loaded with ChR2-EYGP (green channel) can be observed together with the neurobiotin-filled pyramidal neuron recorded in layer 3 of $\mathrm{MPFC}$ (red channel, boxed area). Bottom, Highermagnification image of the neuron shown at top. Maximum intensity projection of series of $Z$ stack of confocal images shows a filled cell with typical pyramidal neuron morphology, including apical dendrite and dendritic spines (inset). $C$, Representative example of a short latency (blue light-evoked) EPSP in an MPFC pyramidal cell during whole-cell current-clamp recording. Gray represents individual responses. Green represents average trace from 30 stimuli. Bottom, Response jitter is consistent with monosynaptic responses in both experimental groups. Each dot represents individual jitter of a recorded neuron from control (Flox: pink dots, $n=8$; red dot, mean \pm SEM) or mutant mice (KO: gray dots, $n=9$; black dot, mean \pm SEM). No significant differences between genotypes were observed (Student's $t$ test, $p=$ 0.51). D, EPSPs evoked by optogenetic stimulation of vHP terminals in $\mathrm{mPFC}$ is reduced in mutant mice (KO) compared with age-matched controls (Flox) during GABAergic blockade. EPSPs were recorded in whole-cell current-clamp configuration with $50 \mu \mathrm{m}$ picrotoxin in the bath. ${ }^{*} p=0.023$ (Student's $t$ test). Error bar indicates mean \pm SEM from 10 cells for flox and 8 for mutant. Each dot represents one recorded neuron (18 neurons from 14 slices from 11 animals).

and which are secondary and result as consequence of a cascade of altered physiological processes. For instance, dopaminergic misbalance can deeply affect PFC information processing and PFC-dependent behaviors (Sawaguchi and Goldman-Rakic, 2017). However, the opposite may also be true, since it has been demonstrated that $\mathrm{mPFC}$ dysfunction alters dopaminergic release in the striatum (Kim et al., 2015). One possible approach to address this problem is to induce controlled pathophysiological conditions and to explore how this affects other physiological processes. In our case, we have introduced a highly specific manipulation (NMDAR ablation) to alter cortical interneuron 
function during early postnatal stage. Among the subsequent pathophysiological alterations evidenced in our model, the reduction in the vHP-mPFC functional connectivity stands out, since it emerges in adulthood, concomitantly with the behavioral abnormalities. Since these alterations cannot solely be attributed to an interneuron dysfunction, we advanced the idea that there is an interaction with developmental factors. This vHP-mPFC hypoconnectivity parallels evidence obtained from SZ patients describing a functional disconnection of the temporal lobe, including hippocampus, and the dorsolateral PFC (Ford et al., 2002; Lawrie et al., 2002; Meyer-Lindenberg et al., 2005; Worbe et al., 2015). Moreover, similar alterations were described for individuals at risk (Benetti et al., 2009) and healthy volunteers carrying candidate risk variants for SZ (Bertolino et al., 2006; Esslinger et al., 2009). Notably, a similar temporal-frontal disconnection has been recently reported in patients affected with antiNMDAR encephalitis (Peer et al., 2017), an antibody-mediated dysfunction of NMDARs that in early stages presents severe neuropsychiatric symptoms, including psychosis and memory deficits. The link between a dysfunction of the vHP-mPFC pathway and SZ-related phenotypes is also well established in different animal models of SZ (Sigurdsson, 2016). Direct intervention on vHP (neonatal vHP lesion model) (Tseng et al., 2009), or indirect by genetic manipulation of SZ-associated genes impacting on development (like the $\mathrm{Df}(16) \mathrm{Al}^{+/-}$mice) (Sigurdsson et al., 2010), may disturb normal HP-PFC connectivity and result in SZlike behavioral phenotypes. Together, these evidence have led the proposal that a vHP-mPFC pathway dysfunction may be a translational pathophysiological intermediate SZ phenotype (Sigurdsson, 2016; Bähner and Meyer-Lindenberg, 2017). Our results not only reinforced the relevance of the vHP-mPFC functional disconnection in SZ, but more importantly, they demonstrate that this alteration can emerge in adulthood as a consequence of an early NMDAR hypofunction in cortical interneurons.

While we probe the impact of interneuron dysfunction on long-distance cortical inputs, by vHP-mPFC pathway activation, we cannot exclude the possibility that other long-distance $\mathrm{mPFC}$ afferents may also be affected. For instance, methylazoxymethanol acetate (MAM) and neonatal vHP lesion SZ models showed altered vHP-mPFC connectivity as well as altered processing of amygdala (Esmaeili and Grace, 2013) or VTA inputs (Guirado et al., 2016). In our case, further studies will be required to unveil the consequences of NMDAR interneuron ablation on other relevant $\mathrm{mPFC}$ afferents. In addition, in our mutant mouse, the NMDAR is ablated in $\mathrm{PV}^{+}$interneurons but is not exclusively restricted to this cell population (Belforte et al., 2010; Nakao et al., 2019). Therefore, the pathophysiological mechanisms described in the present work may also come from other interneuron's subtypes, as it has also been proposed for SZ patients (Dienel and Lewis, 2018).

Because of the postnatal and selective origin of our manipulation, we have been able to demonstrate that some pathophysiological mechanisms reported in SZ patients and SZ animal models (uncoupled firing to $\gamma$ rhythms, altered developmental trajectories, and decreased vHP-mPFC functional connectivity) may be secondary to an interneuron dysfunction or depend on the interaction with developmental factors during adolescence. For instance, NMDAR ablation in $\mathrm{PV}^{+}$interneurons very rapidly impacts on firing and timing of cortical network. In this regard, juvenile asymptomatic mutant mice show uncoordinated, although balanced, augmented $\mathrm{mPFC}$ activity (evidenced by a similar increase in mean firing rate of pyramidal cells and interneurons together with a decrease in neuronal entrainment to local $\gamma$ and distal theta oscillations). Thus, the impact of interneuron manipulation on firing and spike timing emerges before animals go through adolescence and induced changes can be directly attributed to the NMDAR ablation. Instead, the decrease in functional connectivity is present only in adults and cannot be the sole consequence of interneuron dysfunction. However, it is still unclear whether mutant mice go through normal developmental changes, but the result circuit is abnormal due to an altered starting point, or if alternatively, the developmental trajectory is also affected by interneuron dysfunction, leading to an abnormal circuitry function. Our data support the latter, given that alteration in LTD in mPFC becomes evident only in adult mice. This suggests that altered plasticity emerges during adolescence as a result of the ablation of NMDAR in interneurons impacting on mPFC developmental trajectory. Additional experiments will be needed to elucidate whether the mechanisms involved in the enhanced LTD include an altered disynaptic feedforward inhibition or not. A role of $\mathrm{PV}^{+}$interneurons in controlling cortical development is well established, particularly during the first postnatal weeks. Additional evidence also posits that $\mathrm{PV}^{+}$cells, and not pyramidal ones, are a key factor in cortical circuit tuning during adolescence (Morishita et al., 2015).

Recent evidence from large-scale studies in patients with an increased risk for SZ have identified both common and rare genetic variants of glutamatergic signaling cascade genes, including the NMDAR (Kirov et al., 2012). However, evidence linking NMDAR hypofunction to GABAergic interneurons is still limited (Nakazawa et al., 2017). We think that the interneuron dysfunction in SZ may have multiple origins, and altered NMDAR itself may not be the only entry point to impact on $\mathrm{PV}^{+}$interneurons early on postnatal development. For instance, other genes genetically linked to SZ (e.g., $n r g 1$ and erbB4) are key players in the regulation of glutamatergic signaling and its development. For instance, Li et al. (2007) reported that NRG2 stimulation of ErbB4 receptors results in NMDAR internalization in $\mathrm{PV}^{+}$interneurons but not in pyramidal cells. Another study has shown that overexpression of NRG1 Type I, an isoform of NRG1 that has elevated levels in some SZ patients, leads to reduced NMDAR synaptic activity in hippocampal $\mathrm{PV}^{+}$and $\mathrm{CCK}^{+}$interneurons (Kotzadimitriou et al., 2018), reinforcing the idea that, regardless of which NRG-ErBb4 component is affected in SZ, its impact on interneuron's physiology may converge on NMDAR. So, the combined evidence suggests that early postnatal dysfunction of $\mathrm{PV}^{+}$interneurons, arising directly by altered expression of the NMDAR, or indirectly by altered function of associated pathways, may be a key triggering pathophysiological factor for the altered neurodevelopment observed in SZ.

Here we show that mPFC of juvenile mutants exhibits an increased firing occurring in an uncoordinated manner, with neurons firing asynchronously to distal and local oscillations. Thus, we propose that increased uncoordinated firing during adolescence will propitiate anti-Hebbian forms of plasticity, promoting LTD over LTP, and lead to a functional disconnection of the HP-PFC pathway in adulthood. Indeed, treatments enhancing $\mathrm{MPFC}$ LTD can result in electrophysiological hypoconnectivity of long-distance mPFC inputs, presumably from vHP (Banks et al., 2015). In addition, the increased local mPFC GABAergic activity displayed by our mutants during adolescence may actively promote LTD plasticity on pyramidal cells, since it has been shown that local prefrontal GABAergic transmission is 
required for the expression of vHP-induced LTD during adolescence (Caballero et al., 2014b, 2016).

We hypothesize that, in juvenile mutants, the cortical circuit may be operating around an elevated set point for firing rate but preserving the dynamic range of response at population level, leading to a normal capacity of processing local and distal inputs; therefore, no overt SZ phenotype is observed. Once juvenile mutant mice enter adolescence, developmental changes kick in, refining an already altered circuit. Alternatively, interneuron dysfunction, on top of placing the system at an abnormal starting point at the beginning of adolescence, may result in an altered developmental trajectory leading to a disruption in the processing of long-range inputs, like the hippocampal ones. Further studies will be required to establish whether the hyperactivity exhibited by pyramidal neurons results from an alteration in the intrinsic excitability, the excitatory/inhibitory balance at cellular level, or a global network reorganization.

\section{References}

Atallah BV, Bruns W, Carandini M, Scanziani M (2012) Parvalbuminexpressing interneurons linearly transform cortical responses to visual stimuli. Neuron 73:159-170.

Bähner F, Meyer-Lindenberg A (2017) Hippocampal-prefrontal connectivity as a translational phenotype for schizophrenia. Eur Neuropsychopharmacol 27:93-106.

Banks PJ, Burroughs AC, Barker GR, Brown JT, Warburton EC, Bashir ZI (2015) Disruption of hippocampal-prefrontal cortex activity by dopamine D2R-dependent LTD of NMDAR transmission. Proc Natl Acad Sci USA 112:11096-11101.

Bartho P, Hirase H, Monconduit L, Zugaro M, Harris KB (2004) Characterization of neocortical principal cells and interneurons by network interactions and extracellular features. J Neurophysiol 112:1109611101.

Belforte JE, Zsiros V, Sklar ER, Jiang Z, Yu G, Li Y, Quinlan EM, Nakazawa K (2010) Postnatal NMDA receptor ablation in corticolimbic interneurons confers schizophrenia-like phenotypes. Nat Neurosci 13:76-83.

Benetti S, Mechelli A, Picchioni M, Broome M, Williams S, McGuire P (2009) Functional integration between the posterior hippocampus and prefrontal cortex is impaired in both first episode schizophrenia and the at risk mental state. Brain 132:2426-2436.

Bertolino A, Rubino V, Sambataro F, Blasi G, Latorre V, Fazio L, Caforio G, Petruzzella V, Kolachana B, Hariri A, Meyer-Lindenberg A, Nardini M, Weinberger DR, Scarabino T (2006) Prefrontal-hippocampal coupling during memory processing is modulated by COMT val158met genotype. Biol Psychiatry 60:1250-1258.

Bogart LJ, O'Donnell P (2018) Multiple long-range inputs evoke NMDA currents in prefrontal cortex fast-spiking interneurons. Neuropsychopharmacology 43:2101-2108.

Braz BY, Galiñanes GL, Taravini IR, Belforte JE, Murer MG (2015) Altered corticostriatal connectivity and exploration/exploitation imbalance emerge as intermediate phenotypes for a neonatal dopamine dysfunction. Neuropsychopharmacology 40:2576-2587.

Brown AS (2011) The environment and susceptibility to schizophrenia. Prog Neurobiol 93:23-58.

Buzsáki G, Wang XJ (2012) Mechanisms of gamma oscillations. Annu Rev Neurosci 35:203-225.

Caballero A, Flores-Barrera E, Cass DK, Tseng KY (2014a) Differential regulation of parvalbumin and calretinin interneurons in the prefrontal cortex during adolescence. Brain Struct Funct 219:395-406.

Caballero A, Thomases DR, Flores-Barrera E, Cass DK, Tseng KY (2014b) Emergence of GABAergic-dependent regulation of input-specific plasticity in the adult rat prefrontal cortex during adolescence. Psychopharmacology (Berl) 231:1789-1796.

Caballero A, Granberg R, Tseng KY (2016) Mechanisms contributing to prefrontal cortex maturation during adolescence. Neurosci Biobehav Rev 70:4-12.

Casey BJ, Jones RM, Hare TA (2008) The adolescent brain. Ann NY Acad Sci 1124:111-126
Cheetham CE, Fox K (2010) Presynaptic development at L4 to L2/3 excitatory synapses follows different time courses in visual and somatosensory cortex. J Neurosci 1124:111-126.

Cho RY, Konecky RO, Carter CS (2006) Impairments in frontal cortical synchrony and cognitive control in schizophrenia. Proc Natl Acad Sci USA 103:19878-19883.

Clement EA, Richard A, Thwaites M, Ailon J, Peters S, Dickson CT (2008) Cyclic and sleep-like spontaneous alternations of brain state under urethane anaesthesia. PLoS One 3:e2004.

Curtis DR, Duggan AW, Felix D, Johnston GA (1970) GABA, bicuculline and central inhibition. Nature 226:1222-1224.

de Almeida J, Jourdan I, Murer MG, Belforte JE (2013) Refinement of neuronal synchronization with gamma oscillations in the medial prefrontal cortex after adolescence. PLoS One 8:e62978.

Dégenètais E, Thierry AM, Glowinski J, Gioanni Y (2003) Synaptic influence of hippocampus on pyramidal cells of the rat prefrontal cortex: an in vivo intracellular recording study. Cereb Cortex 13:782-792.

Dienel SJ, Lewis DA (2018) Alterations in cortical interneurons and cognitive function in schizophrenia. Neurobiol Dis 131:104208.

Do KQ, Cuenod M, Hensch TK (2015) Targeting oxidative stress and aberrant critical period plasticity in the developmental trajectory to schizophrenia. Schizophr Bull 41:835-846.

Donato F, Rompani SB, Caroni P (2013) Parvalbumin-expressing basket-cell network plasticity induced by experience regulates adult learning. Nature 504:272-276

Esmaeili B, Grace AA (2013) Afferent drive of medial prefrontal cortex by hippocampus and amygdala is altered in MAM-treated rats: evidence for interneuron dysfunction. Neuropsychopharmacology 38:1871-1880.

Espíndola SL, Damianich A, Alvarez RJ, Sartor M, Belforte JE, Ferrario JE, Gallo JM, Avale ME (2018) Modulation of tau isoforms imbalance precludes tau pathology and cognitive decline in a mouse model of tauopathy. Cell Rep 23:709-715.

Esslinger C, Walter H, Kirsch P, Erk S, Schnell K, Arnold C, Haddad L, Mier D, Boberfeld CO, Von Raab K, Witt SH, Rietschel M, Cichon S, MeyerLindenberg A (2009) Neural mechanisms of a genome-wide supported psychosis variant. Science 324:605.

Ford JM, Mathalon DH, Whitfield S, Faustman WO, Roth WT (2002) Reduced communication between frontal and temporal lobes during talking in schizophrenia. Biol Psychiatry 51:485-492.

Fuchs EC, Zivkovic AR, Cunningham MO, Middleton S, Lebeau FE, Bannerman DM, Rozov A, Whittington MA, Traub RD, Rawlins JN, Monyer H (2007) Recruitment of parvalbumin-positive interneurons determines hippocampal function and associated behavior. Neuron 53:591-604.

Gabbott P, Headlam A, Busby S (2002) Morphological evidence that CA1 hippocampal afferents monosynaptically innervate PV-containing neurons and NADPH-diaphorase reactive cells in the medial prefrontal cortex (Areas 25/32) of the rat. Brain Res 946:314-322.

Gervasoni D, Lin SC, Ribeiro S, Soares ES, Pantoja J, Nicolelis MA (2004) Global forebrain dynamics predict rat behavioral states and their transitions. J Neurosci 24:11137-11147.

Gigg J, Tan AM, Finch DM (1994) Glutamatergic hippocampal formation projections to prefrontal cortex in the rat are regulated by GABAergic inhibition and show convergence with glutamatergic projections from the limbic thalamus. Hippocampus 4:189-198.

Gomes FV, Rincón-Cortés M, Grace AA (2016) Adolescence as a period of vulnerability and intervention in schizophrenia: insights from the MAM model. Neurosci Biobehav Rev 70:260-270.

Guirado R, Umemori J, Sipilä P, Castrén E (2016) Evidence for competition for target innervation in the medial prefrontal cortex. Cereb Cortex 26:1287-1294.

Gurden H, Takita M, Jay TM (2000) Essential role of D1 but not D2 receptors in the NMDA receptor-dependent long-term potentiation at hippocampal-prefrontal cortex synapses in vivo. J Neurosci 20:RC106.

Haider B, McCormick DA (2009) Rapid neocortical dynamics: cellular and network mechanisms. Neuron 62:171-189.

Hasenstaub A, Shu Y, Haider B, Kraushaar U, Duque A, McCormick DA (2005) Inhibitory postsynaptic potentials carry synchronized frequency information in active cortical networks. Neuron 47:423-435.

Hazan L, Zugaro M, Buzsáki G (2006) Klusters, NeuroScope, NDManager: a free software suite for neurophysiological data processing and visualization. J Neurosci Methods 155:207-216. 
Hensch TK, Fagiolini M, Mataga N, Stryker MP, Baekkeskov S, Kash SF (1998) Local GABA circuit control of experience-dependent plasticity in developing visual cortex. Science 282:1504-1508.

Hoenig JM, Heisey DM (2001) The abuse of power: the pervasive fallacy of power calculations for data analysis. Am Stat 55:19-24.

Hoftman GD, Lewis DA (2011) Postnatal developmental trajectories of neural circuits in the primate prefrontal cortex: identifying sensitive periods for vulnerability to schizophrenia. Schizophr Bull 37:493-503.

Insel T, Cuthbert B, Garvey M, Heinssen R, Pine D, Quinn K, Sanislow C, Wang P (2010) Research Domain Criteria (RDoC): toward a new classification framework for research on mental disorders. Am J Psychiatry 167:748-751.

Jiang Z, Rompala GR, Zhang S, Cowell RM, Nakazawa K (2013) Social isolation exacerbates schizophrenia-like phenotypes via oxidative stress in cortical interneurons. Biol Psychiatry 73:1024-1034.

Kannan G, Sawa A, Pletnikov MV (2013) Mouse models of gene-environment interactions in schizophrenia. Neurobiol Dis 57:5-11.

Kasanetz F, Riquelme LA, Della-Maggiore V, O'Donnell P, Murer MG (2008) Functional integration across a gradient of corticostriatal channels controls UP state transitions in the dorsal striatum. Proc Natl Acad Sci USA 105:8124-8129.

Keifman E, Ruiz-DeDiego I, Pafundo DE, Paz RM, Solís O, Murer MG, Moratalla R (2019) Optostimulation of striatonigral terminals in substantia nigra induces dyskinesia that increases after L-DOPA in a mouse model of Parkinson's disease. Br J Pharmacol 176:2146-2161.

Kim IH, Rossi MA, Aryal DK, Racz B, Kim N, Uezu A, Fan W, Wetsel WC, Weinberg RJ, Yin H, Soderling SH (2015) Spine pruning drives antipsychotic-sensitive locomotion via circuit control of striatal dopamine. Nat Neurosci 18:883-891.

Kirov G, Pocklington AJ, Holmans P, Ivanov D, Ikeda M, Ruderfer D, Moran J, Chambert K, Toncheva D, Georgieva L, Grozeva D, Fjodorova M, Wollerton R, Rees E, Nikolov I, van de Lagemaat LN, Bayés À, Fernandez E, Olason PI, Böttcher Y, et al. (2012) De novo $\mathrm{CNV}$ analysis implicates specific abnormalities of postsynaptic signalling complexes in the pathogenesis of schizophrenia. Mol Psychiatry 17:142-153.

Kotzadimitriou D, Nissen W, Paizs M, Newton K, Harrison PJ, Paulsen O, Lamsa K (2018) Neuregulin 1 type I overexpression is associated with reduced NMDA receptor-mediated synaptic signaling in hippocampal interneurons expressing PV or CCK. eNeuro 5: ENEURO.0418-17.2018.

Kuhlman SJ, Olivas ND, Tring E, Ikrar T, Xu X, Trachtenberg JT (2013) A disinhibitory microcircuit initiates critical-period plasticity in the visual cortex. Nature 501:543-546.

Laroche S, Jay TM, Thierry AM (1990) Long-term potentiation in the prefrontal cortex following stimulation of the hippocampal CA1/subicular region. Neurosci Lett 114:184-190.

Lawrie SM, Buechel C, Whalley HC, Frith CD, Friston KJ, Johnstone EC (2002) Reduced frontotemporal functional connectivity in schizophrenia associated with auditory hallucinations. Biol Psychiatry 51:1008-1011.

Lee SH, Kwan AC, Zhang S, Phoumthipphavong V, Flannery JG, Masmanidis SC, Taniguchi H, Huang ZJ, Zhang F, Boyden ES, Deisseroth K, Dan Y (2012) Activation of specific interneurons improves V1 feature selectivity and visual perception. Nature 488:379-383.

Lewis DA, Levitt P (2002) Schizophrenia as a disorder of neurodevelopment. Annu Rev Neurosci 25:409-432.

Li B, Woo RS, Mei L, Malinow R (2007) The neuregulin-1 receptor ErbB4 controls glutamatergic synapse maturation and plasticity. Neuron 54:583-597.

Maggi CA, Meli A (1986) Suitability of urethane anesthesia for physiopharmacological investigations: 3 . Other systems and conclusions. Experientia 42:531-537.

Marek R, Jin J, Goode TD, Giustino TF, Wang Q, Acca GM, Holehonnur R, Ploski JE, Fitzgerald PJ, Lynagh T, Lynch JW, Maren S, Sah P (2018) Hippocampus-driven feed-forward inhibition of the prefrontal cortex mediates relapse of extinguished fear. Nat Neurosci 21:384-392.

Meletis K, Siegle JH, Cardin JA, Futai K, Ru C, Carlén M, Vierling-Claassen D, Rühlmann C, Jones SR, Deisseroth K, Sheng M, Moore CI, Tsai LH (2011) A critical role for NMDA receptors in parvalbumin interneurons for gamma rhythm induction and behavior. Mol Psychiatry 17:537-548.
Meyer-Lindenberg AS, Olsen RK, Kohn PD, Brown T, Egan MF, Weinberger DR, Berman KF (2005) Regionally specific disturbance of dorsolateral prefrontal-hippocampal functional connectivity in schizophrenia. Arch Gen Psychiatry 62:379-386.

Morishita H, Cabungcal JH, Chen Y, Do KQ, Hensch TK (2015) Prolonged period of cortical plasticity upon redox dysregulation in fast-spiking interneurons. Biol Psychiatry 78:396-402.

Mouri A, Noda Y, Enomoto T, Nabeshima T (2007) Phencyclidine animal models of schizophrenia: approaches from abnormality of glutamatergic neurotransmission and neurodevelopment. Neurochem Int 51:173184.

Nakao K, Jeevakumar V, Jiang SZ, Fujita Y, Diaz NB, Annan CA, Jaunarajs KL, Hashimoto K, Belforte JE, Nakazawa K (2019) Schizophrenia-like dopamine release abnormalities in a mouse model of NMDA receptor hypofunction. Schizophr Bull 45:138-147.

Nakazawa K, Jeevakumar V, Nakao K (2017) Spatial and temporal boundaries of NMDA receptor hypofunction leading to schizophrenia. NPJ Schizophr 3:7.

Packer AM, Yuste R (2011) Dense, unspecific connectivity of neocortical parvalbumin-positive interneurons: a canonical microcircuit for inhibition? J Neurosci 31:13260-13271.

Pafundo DE, Miyamae T, Lewis DA, Gonzalez-Burgos G (2018) Presynaptic effects of N-methyl-D-aspartate receptors enhance parvalbumin cellmediated inhibition of pyramidal cells in mouse prefrontal cortex. Biol Psychiatry 84:460-470.

Paus TT, Keshavan M, Giedd JN, Keshavan M, Paus TT (2008) Why do many psychiatric disorders emerge during adolescence? Nat Rev Neurosci 9:947-957.

Peer M, Prüss H, Ben-Dayan I, Paul F, Arzy S, Finke C (2017) Functional connectivity of large-scale brain networks in patients with anti-NMDA receptor encephalitis: an observational study. Lancet Psychiatry 4:768774 .

Pouille F, Marin-Burgin A, Adesnik H, Atallah BV, Scanziani M (2009) Input normalization by global feedforward inhibition expands cortical dynamic range. Nat Neurosci 12:1577-1585.

Pouille F, Scanziani M (2001) Enforcement of temporal fidelity in pyramidal cells by somatic feed-forward inhibition. Science 293:1159-1163.

Pozzi L, Dorocic IP, Wang X, Carlén M, Meletis K (2014) Mice lacking NMDA receptors in parvalbumin neurons display normal depressionrelated behavior and response to antidepressant action of NMDAR antagonists. PLoS One 9:e83879.

Rapoport JL, Giedd JN, Gogtay N (2012) Neurodevelopmental model of schizophrenia: update 2012. Mol Psychiatry 17:1228-1238.

Sawaguchi T, Goldman-Rakic PS (2017) The role of D1-dopamine receptor in working memory: local injections of dopamine antagonists into the prefrontal cortex of rhesus monkeys performing an oculomotor delayedresponse task. J Neurophysiol. 71:515-528.

Sigurdsson T (2016) Neural circuit dysfunction in schizophrenia: insights from animal models. Neuroscience 321:42-65.

Sigurdsson T, Stark KL, Karayiorgou M, Gogos JA, Gordon JA (2010) Impaired hippocampal-prefrontal synchrony in a genetic mouse model of schizophrenia. Nature 464:763-767.

Sirota A, Jones MW, Montgomery S, Wilson MA, Fujisawa S, Isomura Y, Zugaro M, Buzsáki G (2008) Entrainment of neocortical neurons and gamma oscillations by the hippocampal theta rhythm. Neuron 60:683697.

Somogyi P, Klausberger T (2005) Defined types of cortical interneurone structure space and spike timing in the hippocampus. J Physiol $562: 9-26$.

Spear LP (2000) The adolescent brain and age-related behavioral manifestations. Neurosci Biobehav Rev 24:417-463.

Spencer KM, Nestor PG, Niznikiewicz MA, Salisbury DF, Shenton ME, McCarley RW (2003) Abnormal neural synchrony in schizophrenia. J Neurosci 23:7407-7411.

Steriade M, Timofeev I, Grenier F (2001) Natural waking and sleep states: a view from inside neocortical neurons. J Neurophysiol 85: 1969-1985.

Thierry AM, Gioanni Y, Dégénétais E, Glowinski J (2000) Hippocampo-prefrontal cortex pathway: anatomical and electrophysiological characteristics. Hippocampus 10:411-419. 
Tierney PL, Dégenètais E, Thierry AM, Glowinski J, Gioanni Y (2004) Influence of the hippocampus on interneurons of the rat prefrontal cortex. Eur J Neurosci 20:514-524.

Timofeev I, Grenier F, Steriade M (2000) Impact of intrinsic properties and synaptic factors on the activity of neocortical networks in vivo. J Physiol Paris 94:343-355.

Tseng KY, Chambers RA, Lipska BK (2009) The neonatal ventral hippocampal lesion as a heuristic neurodevelopmental model of schizophrenia. Behav Brain Res 204:295-305.

Tsuang MT, Stone WS, Faraone SV (2001) Genes, environment and schizophrenia. Br J Psychiatry 178:s18-s24.

Uhlhaas PJ, Singer W (2010) Abnormal neural oscillations and synchrony in schizophrenia. Nat Rev Neurosci 11:100-113. van Os J, Kapur S (2009) Schizophrenia. Lancet 374:635-645.

van Os J, Kenis G, Rutten BPF (2010) The environment and schizophrenia. Nature 468:203-212.

Wehr M, Zador AM (2003) Balanced inhibition underlies tuning and sharpens spike timing in auditory cortex. Nature 426:442-446.

Weinberger DR (1987) Implications of normal brain development for the pathogenesis of schizophrenia. Arch Gen Psychiatry 44:660669.

Worbe Y, Marrakchi-Kacem L, Lecomte S, Valabregue R, Poupon F, Guevara P, Tucholka A, Mangin JF, Vidailhet M, Lehericy S, Hartmann A, Poupon C (2015) Altered structural connectivity of cortico-striato-pallido-thalamic networks in Gilles de la Tourette syndrome. Brain 138:472-482. 